\title{
Synthesis of a tetraphenyl-substituted dihydropentalene and its alkali metal hydropentalenide and pentalenide complexes
}

Stuart M. Boyt, ${ }^{a}$ Niko A. Jenek, ${ }^{a}$ Hugh J. Sanderson, ${ }^{a}$ Gabriele Kociok-Köhn, ${ }^{b}$ Ulrich Hintermair*a,c

a) Department of Chemistry, University of Bath, Claverton Down, Bath BA2 7AY, UK.

b) Material and Chemical Characterisation Facility, University of Bath, Claverton Down, Bath BA2 7AY, UK.

c) Centre for Sustainable \& Circular Technologies, University of Bath, Claverton Down, Bath, BA2 7AY, UK.

*u.hintermair@bath.ac.uk

\section{SUPPORTING INFORMATION}




\section{Table of Contents}

1. Alternative syntheses of 1,3,4,6-tetraphenyl-1,2-dihydropentalene $\left(1,3,4,6-\mathrm{Pn}_{4} \mathrm{PnH}_{2}\right) \ldots \ldots \ldots \ldots . . . . .3$

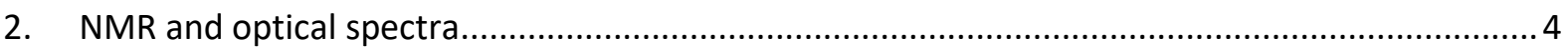

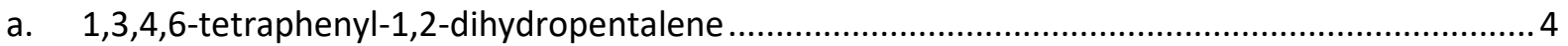

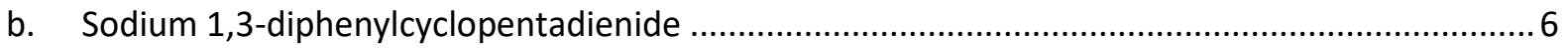

c. Lithium 1,3,4,6-tetraphenylhydropentalenide ...................................................................... 8

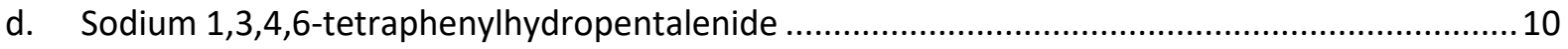

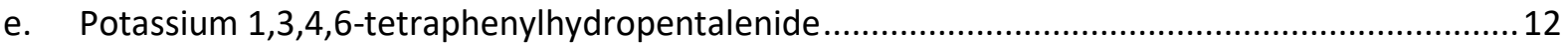

f. Lithium potassium 1,3,4,6-tetraphenylpentalenide ................................................................ 13

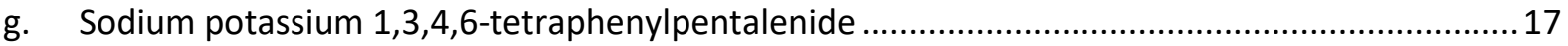

h. Sodium lithium 1,3,4,6-tetraphenylpentalenide ................................................................. 18

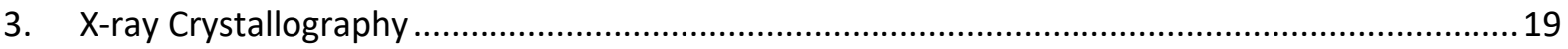

a. Crystal data and structure refinement for 1,3,4,6-tetraphenyldihydropentalene.......................20

b. Crystal data and structure refinement for potassium 1,3,4,6-tetraphenylhydropentalenide ......21

c. Crystal data and structure refinement for dilithium 1,3,4,6-tetraphenylpentalenide .................22

d. Crystal data and structure refinement for disodium 1,3,4,6-tetraphenylpentalenide .................23

e. Crystal data and structure refinement for thf-coordinated dipotassium 1,3,4,6-

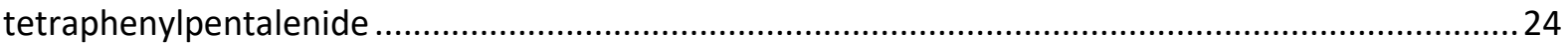

f. Crystal data and structure refinement for pyridine-coordinated dipotassium 1,3,4,6-

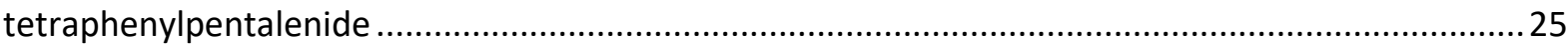




\section{Alternative syntheses of 1,3,4,6-tetraphenyl-1,2-dihydropentalene $\left(1,3,4,6-\mathrm{Pn}_{4} \mathrm{PnH}_{2}\right)$}

A. 1,4-diphenyl-1,3-cyclopentadiene $(1.00 \mathrm{~g}, 4.58 \mathrm{mmol})$ and 1,3-diphenylprop-2-en-1-one (1.05 g, $5.04 \mathrm{mmol}$ ) were dissolved in $20 \mathrm{ml}$ dry methanol and $20 \mathrm{ml}$ dry toluene under argon. To this was added a solution of potassium fluoride $(0.47 \mathrm{~g}, 4.58 \mathrm{mmol})$ and 18 -crown-6 $(1.20 \mathrm{~g}, 4.58 \mathrm{mmol})$ in 10 $\mathrm{ml}$ dry toluene and $10 \mathrm{ml}$ dry methanol. The resulting solution was stirred for 48 hours at $70{ }^{\circ} \mathrm{C}$. The solvent was removed under reduced pressure. In air the crude material was dissolved in $150 \mathrm{ml}$ diethyl ether and washed with $3 \times 150 \mathrm{ml}$ water and $150 \mathrm{ml}$ brine. The ether fraction was dried over $\mathrm{MgSO}_{4}$ and the solvent the solvent removed reduced pressure. The fraction was then filtered through silica using 1:1 diethyl ether/hexane as the eluent, and the solvent removed under reduced pressure. The resulting dark red solid was recrystallized from ethanol to give a dark red crystalline solid $(0.77 \mathrm{~g}, 1.88$ $\mathrm{mmol}, 41 \%)$.

B. 1,4-diphenyl-1,3-cyclopentadiene $(1.00 \mathrm{~g}, 4.58 \mathrm{mmol})$ and 1,3-diphenylprop-2-en-1-one (1.05 g, $5.04 \mathrm{mmol}$ ) were dissolved in $20 \mathrm{ml}$ dry methanol under argon. To this was added sodium methoxide $(0.25 \mathrm{~g}, 4.58 \mathrm{mmol})$ in $20 \mathrm{ml}$ dry methanol, and to the resulting solution was stirred for 48 hours at $70{ }^{\circ} \mathrm{C}$. The solvent was removed under reduced pressure. In air the crude material was dissolved in $150 \mathrm{ml}$ diethyl ether and washed with $3 \times 150 \mathrm{ml}$ water and $150 \mathrm{ml}$ brine. The ether fraction was dried over $\mathrm{MgSO}_{4}$ and the solvent the solvent removed reduced pressure. The fraction was then filtered through silica using 1:1 diethyl ether/hexane as the eluent, and the solvent removed under reduced pressure. The resulting dark red solid was recrystallized from ethanol to give a dark red crystalline solid $(1.21 \mathrm{~g}, 2.98 \mathrm{mmol}, 65 \%)$.

C. 1,4-diphenyl-1,3-cyclopentadiene $(1.00 \mathrm{~g}, 4.58 \mathrm{mmol})$ and 1,3-diphenylprop-2-en-1-one (1.05 g, $5.04 \mathrm{mmol}$ ) were dissolved in $20 \mathrm{ml}$ dry THF under argon. To this was added sodium tert-butoxide $(0.44 \mathrm{~g}, 4.58 \mathrm{mmol})$ in $20 \mathrm{ml}$ dry THF, and to the resulting solution was stirred for 44 hours at $70^{\circ} \mathrm{C}$. The solvent was removed under reduced pressure. In air the crude material was dissolved in $150 \mathrm{ml}$ diethyl ether and washed with $3 \times 150 \mathrm{ml}$ water and $150 \mathrm{ml}$ brine. The ether fraction was dried over $\mathrm{MgSO}_{4}$ and the solvent the solvent removed reduced pressure. The fraction was then filtered through silica using 1:1 diethyl ether/hexane as the eluent, and the solvent removed under reduced pressure. The resulting dark red solid was recrystallized from ethanol to give a dark red crystalline solid $(0.99 \mathrm{~g}$, $2.43 \mathrm{mmol}, 53 \%)$.

D. 1,3,6-Triphenylfulvene $(0.50 \mathrm{~g}, 1.63 \mathrm{mmol})$ and acetophenone $(0.21 \mathrm{ml}, 1.80 \mathrm{mmol})$ were dissolved in $10 \mathrm{ml}$ methanol and $10 \mathrm{ml}$ toluene under argon, and to this was added pyrrolidine $(0.14 \mathrm{ml}, 1.63$ mmol). The resulting mixture was stirred at $70{ }^{\circ} \mathrm{C}$ for 42 hours. The solvent was removed under reduced pressure. In air the crude material was dissolved in $150 \mathrm{ml}$ diethyl ether and washed with $3 \mathrm{x}$ $150 \mathrm{ml}$ water and $150 \mathrm{ml}$ brine. The ether fraction was dried over $\mathrm{MgSO}_{4}$ and the solvent the solvent removed reduced pressure. The fraction was then filtered through silica using 1:1 diethyl ether/hexane as the eluent, and the solvent removed under reduced pressure. The resulting dark red solid was recrystallized from ethanol to give a dark red crystalline solid $(0.48 \mathrm{~g}, 1.17 \mathrm{mmol}, 72 \%)$. 


\section{NMR and optical spectra}

a. 1,3,4,6-tetraphenyl-1,2-dihydropentalene

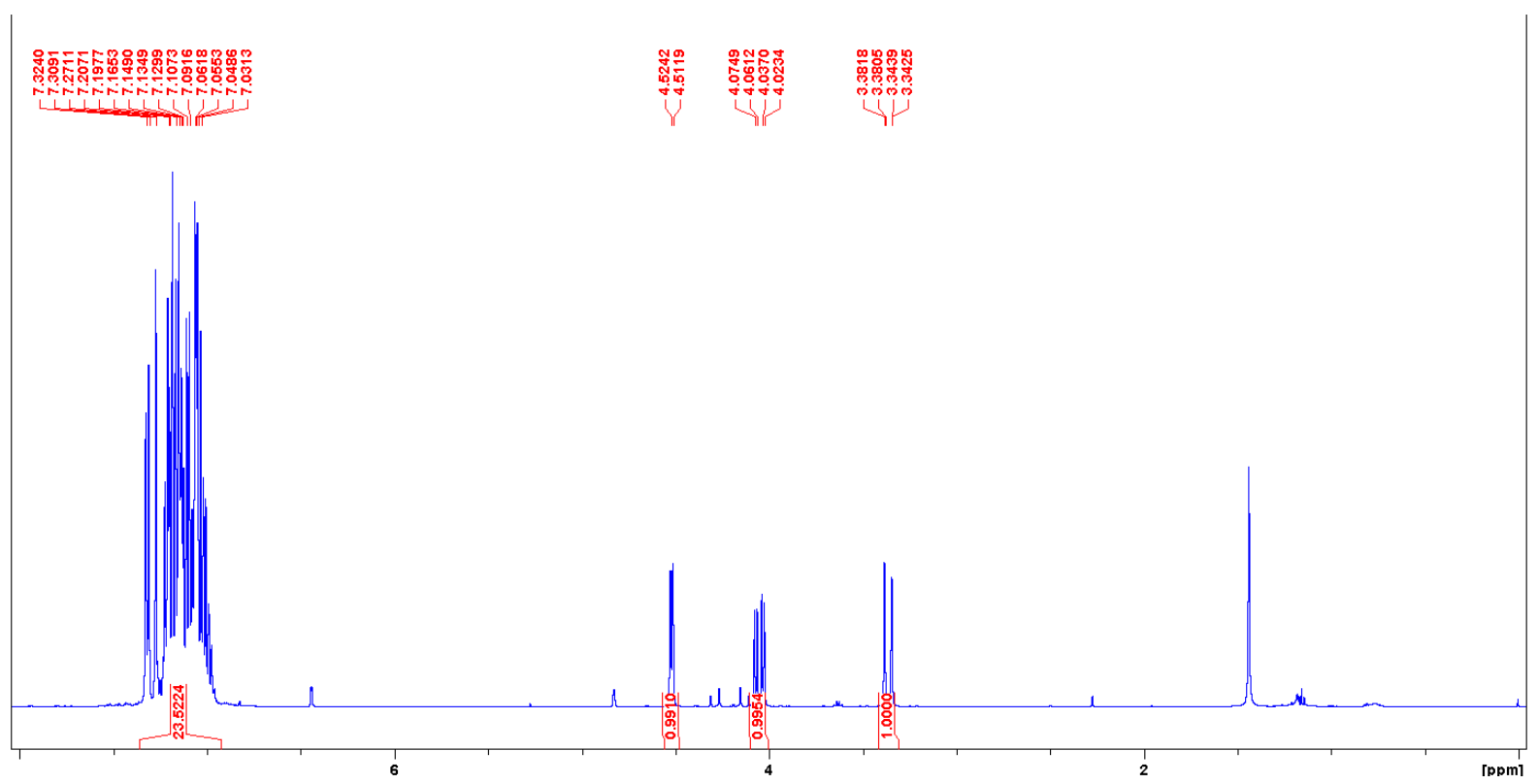

Figure S1: ${ }^{1} \mathrm{H}-\mathrm{NMR}$ spectrum of $\mathrm{Ph}_{4} \mathrm{PnH}_{2}\left(500 \mathrm{MHz}, \mathrm{CDCl}_{3}, 298 \mathrm{~K}\right)$

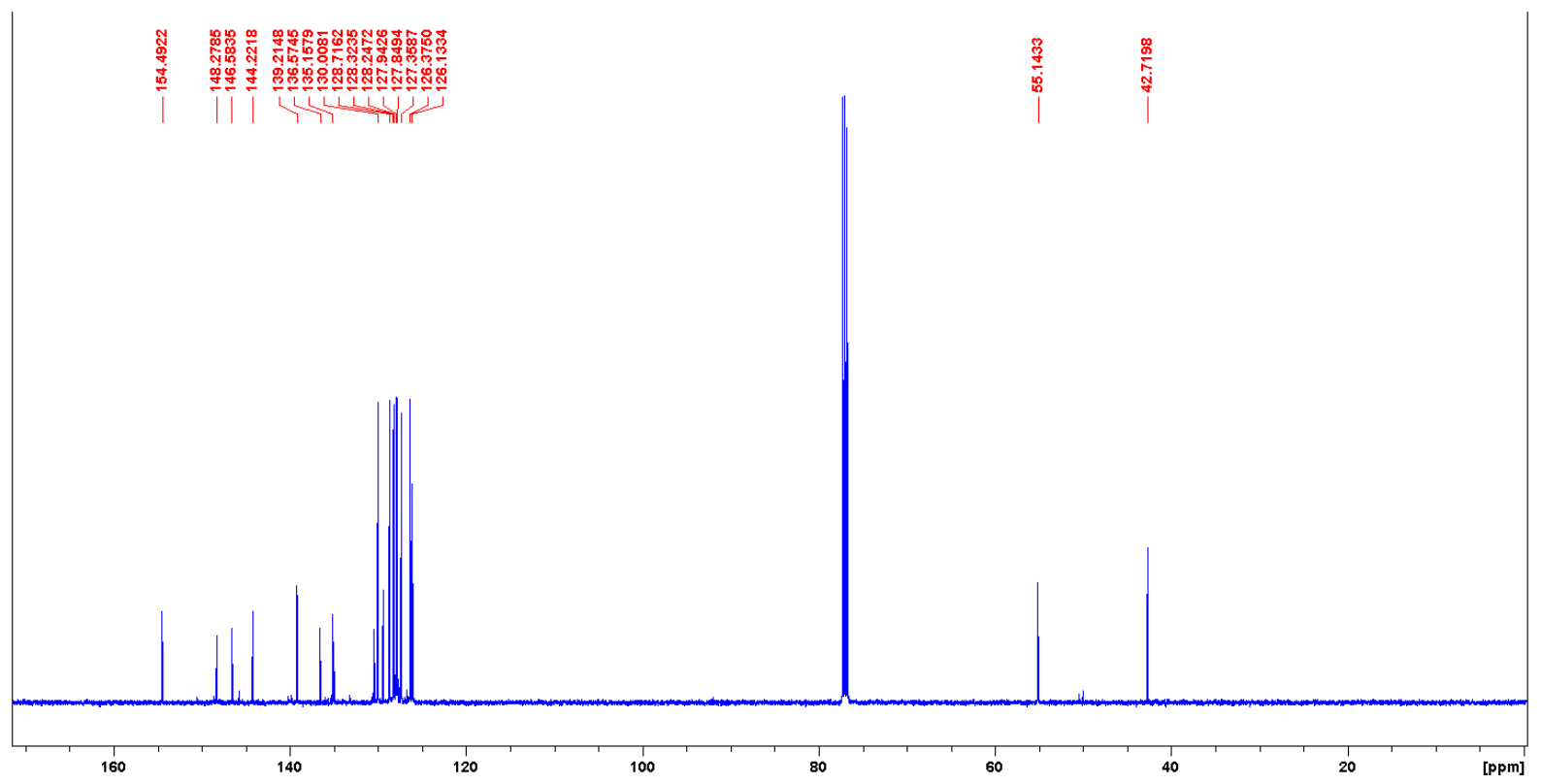

Figure S2: ${ }^{13} \mathrm{C}\left\{{ }^{1} \mathrm{H}\right\}-\mathrm{NMR}$ spectrum of $\mathrm{Ph}_{4} \mathrm{PnH}_{2}\left(125 \mathrm{MHz}, \mathrm{CDCl}_{3}, 298 \mathrm{~K}\right)$ 


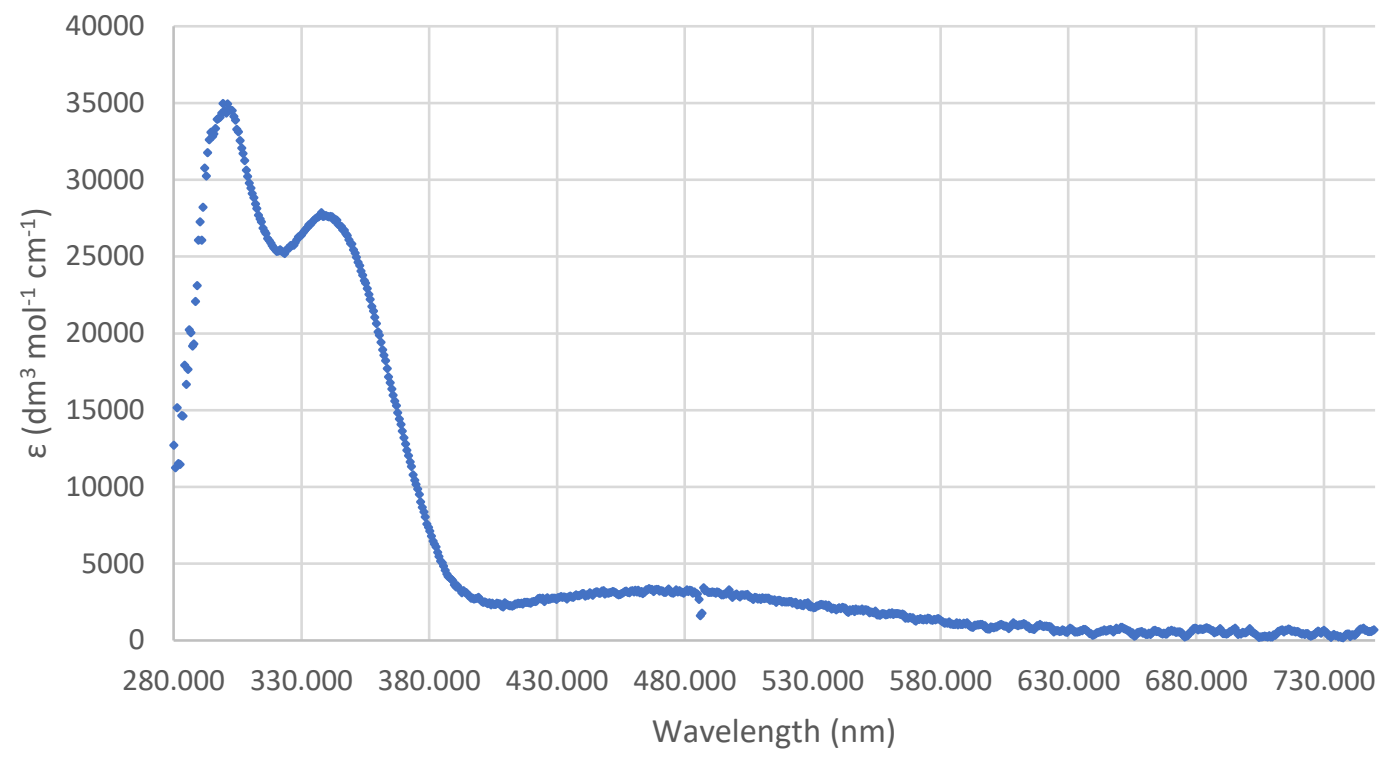

Figure S3: UV/Vis spectrum of $\mathrm{Ph}_{4} \mathrm{PnH}_{2}$ in THF at $293 \mathrm{~K}$ 


\section{b. Sodium 1,3-diphenylcyclopentadienide}

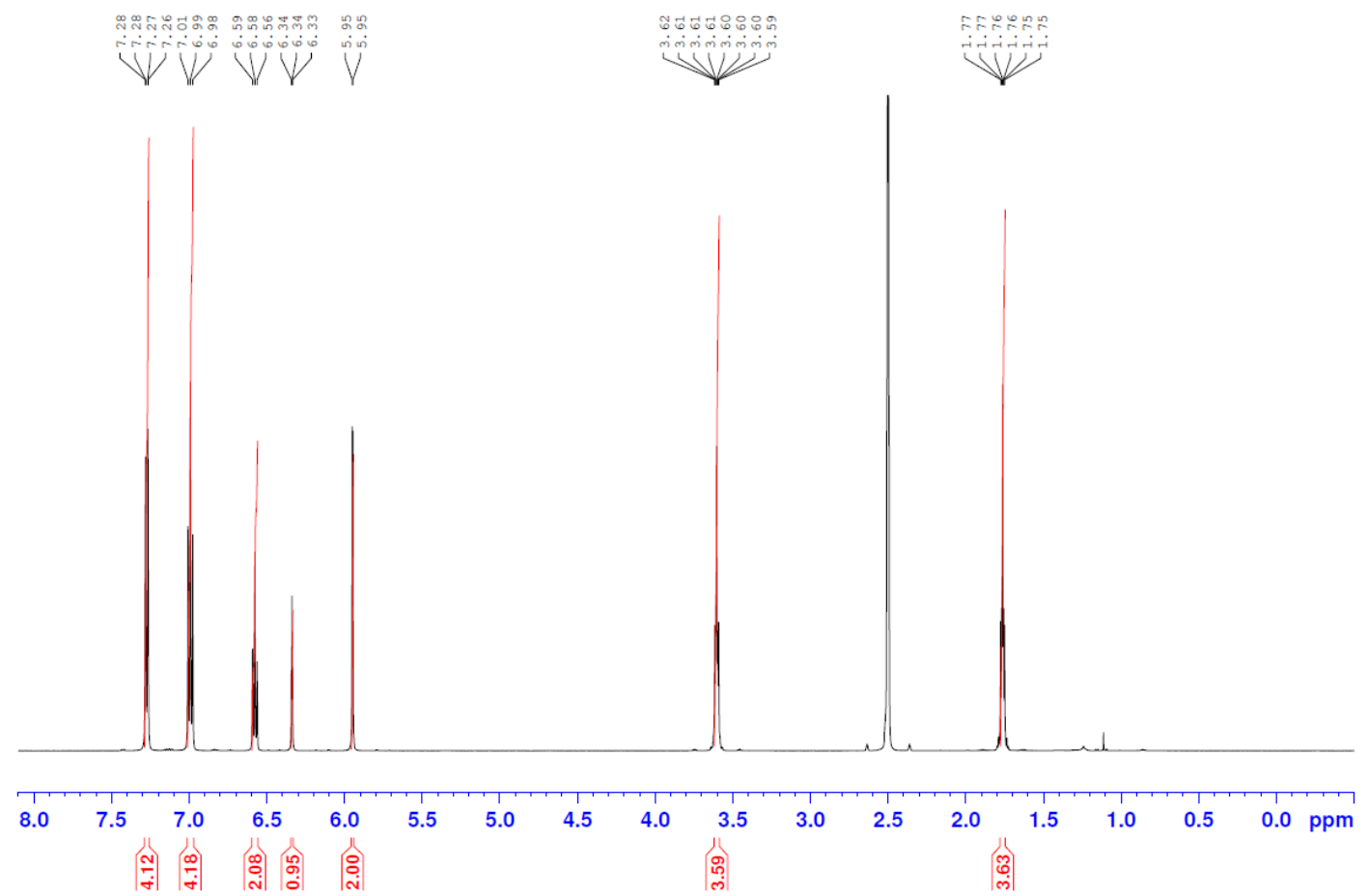

Figure S4: Quantitative ${ }^{1} \mathrm{H}-\mathrm{NMR}$ spectrum of $\mathrm{Na}(\mathrm{THF})_{0.9}\left[\mathrm{CpPh}_{2}\right]\left(500 \mathrm{MHz}, \mathrm{d}_{6}-\mathrm{DMSO}, 298 \mathrm{~K}\right)$

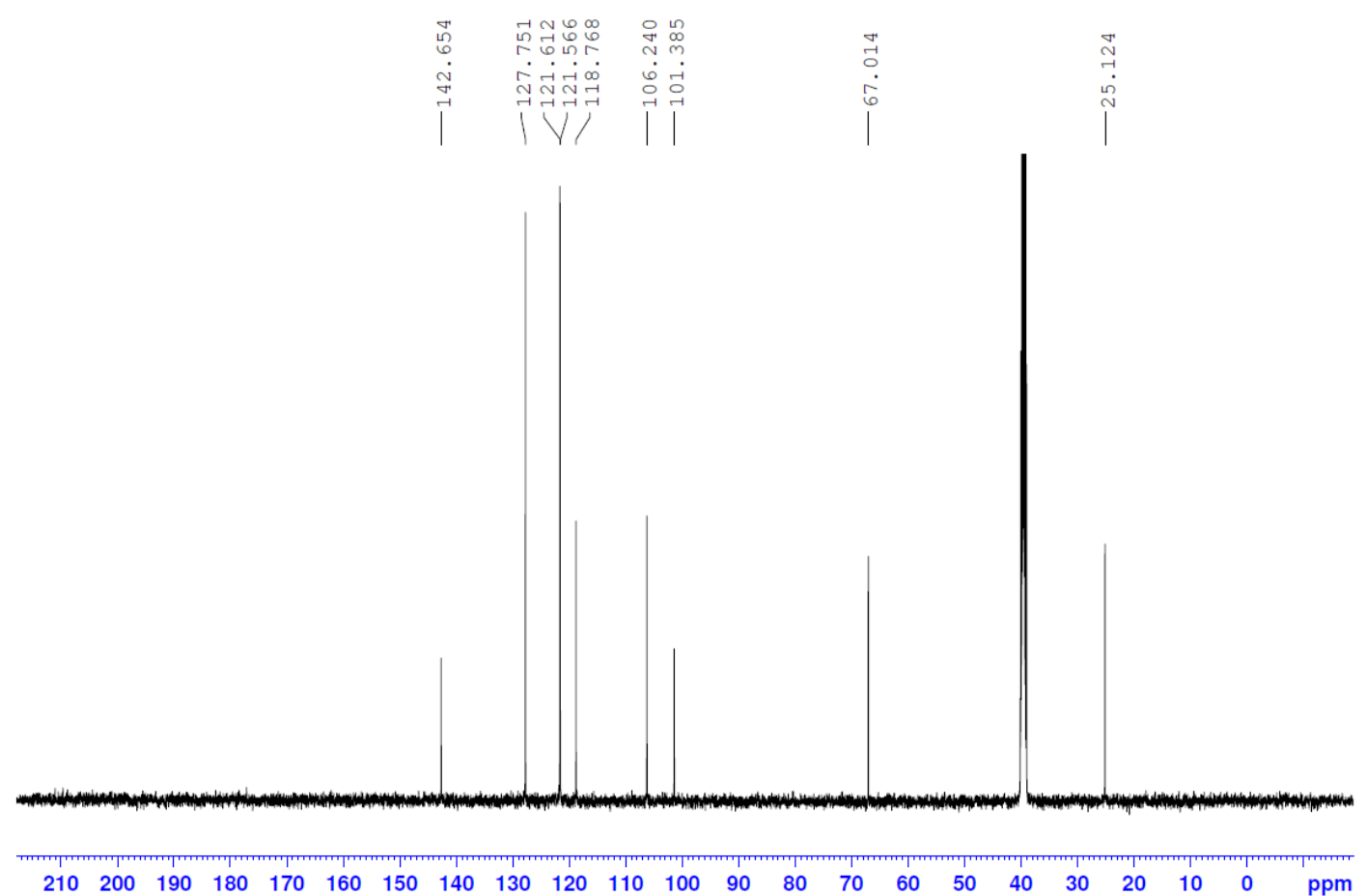

Figure $\mathrm{S} 5:{ }^{13} \mathrm{C}\left\{{ }^{1} \mathrm{H}\right\}-\mathrm{NMR}$ spectrum of $\mathrm{Na}(\mathrm{THF})_{0.9}\left[\mathrm{CpPh}_{2}\right]\left(125 \mathrm{MHz}, \mathrm{d}_{6}-\mathrm{DMSO}, 298 \mathrm{~K}\right)$ 


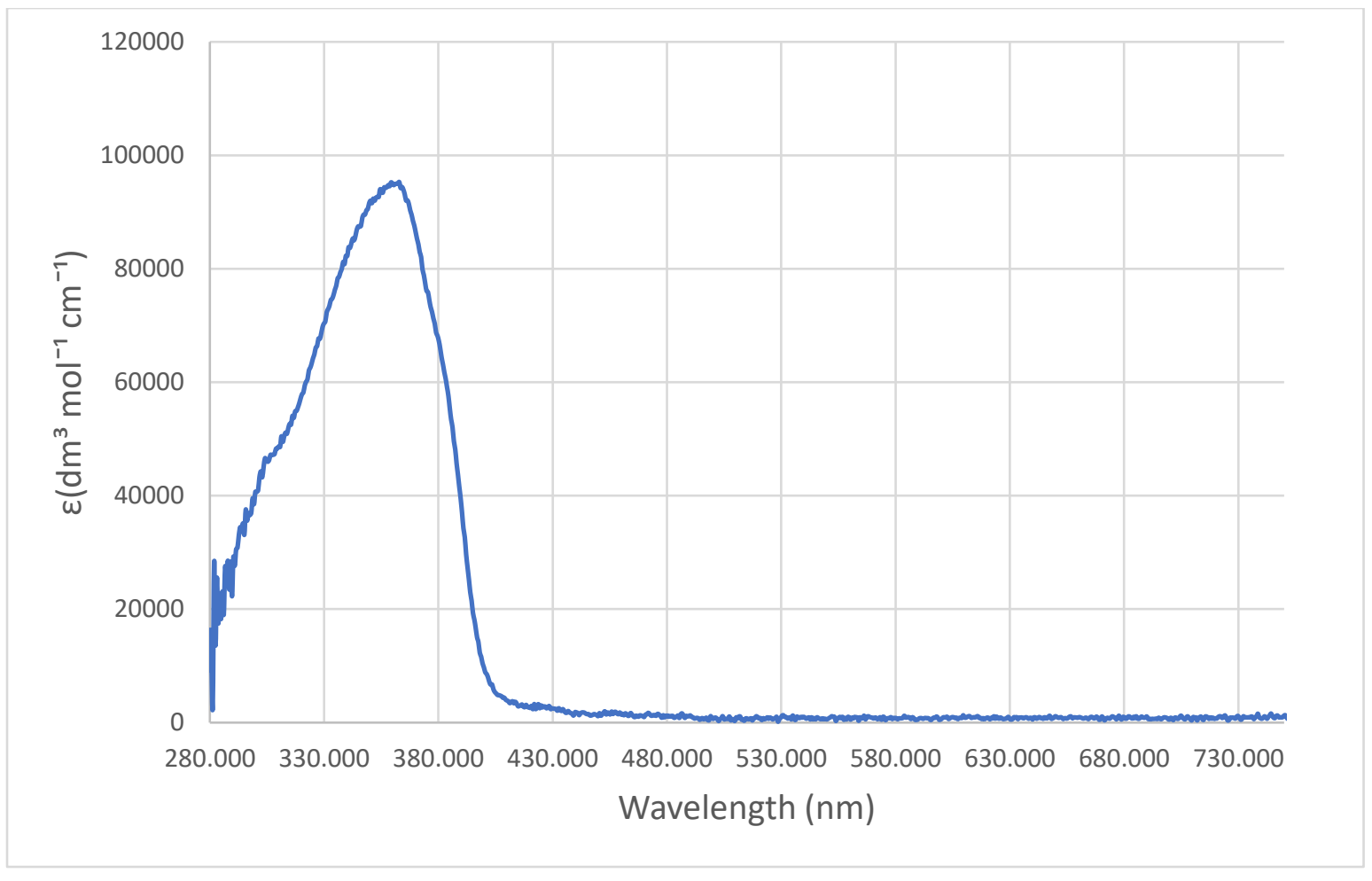

Figure S6: UV/Vis spectrum of $\mathrm{Na}(\mathrm{THF})_{0.9}\left[\mathrm{CpPh}_{2}\right]$ in THF at $293 \mathrm{~K}$ 
c. Lithium 1,3,4,6-tetraphenylhydropentalenide

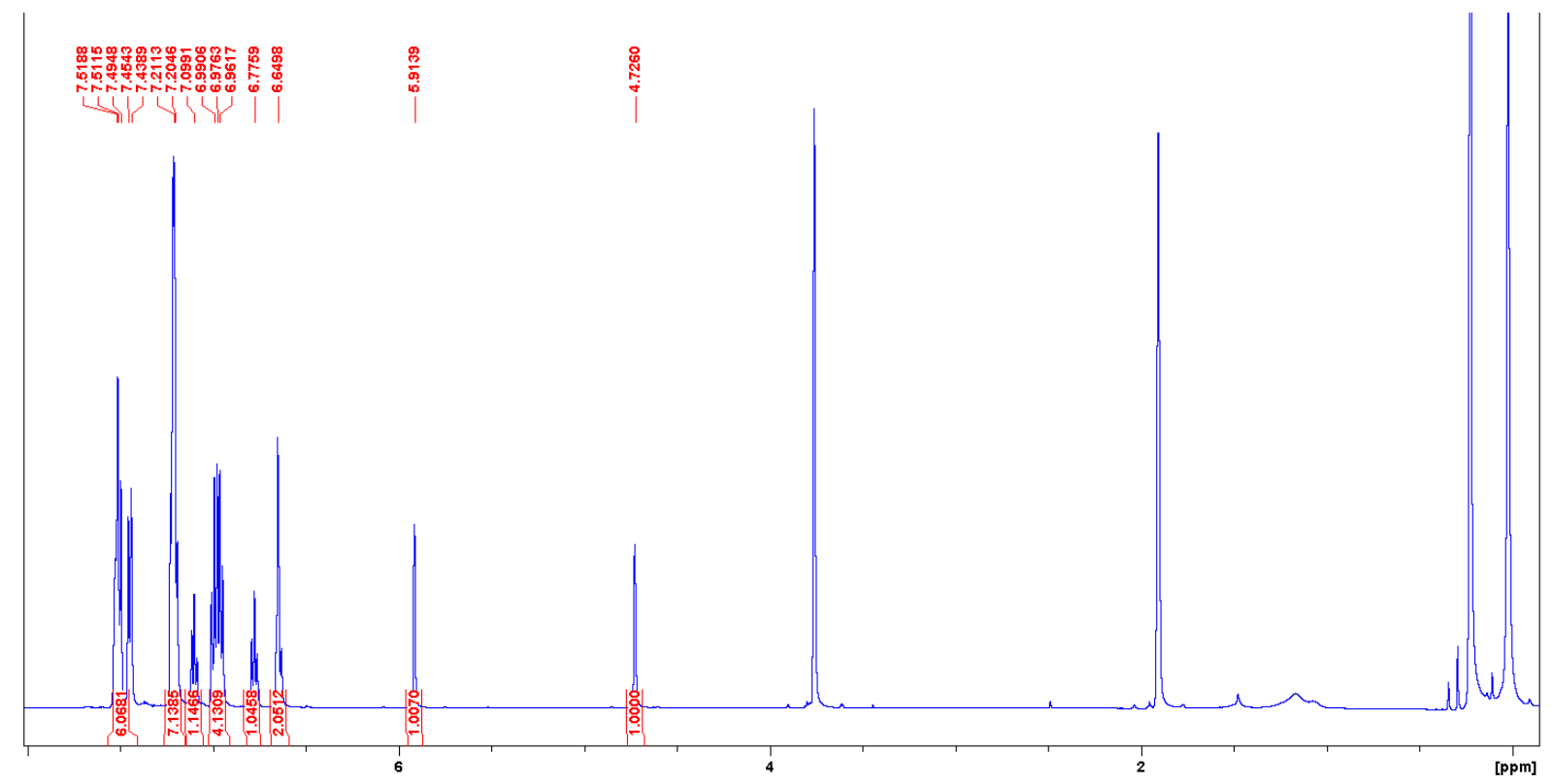

Figure S7: ${ }^{1} \mathrm{H}-\mathrm{NMR}$ spectrum of $\mathrm{Li}\left[\mathrm{Ph}_{4} \mathrm{PnH}\right]\left(500 \mathrm{MHz}, \mathrm{d}_{8}-\mathrm{THF}, 298 \mathrm{~K}\right)$

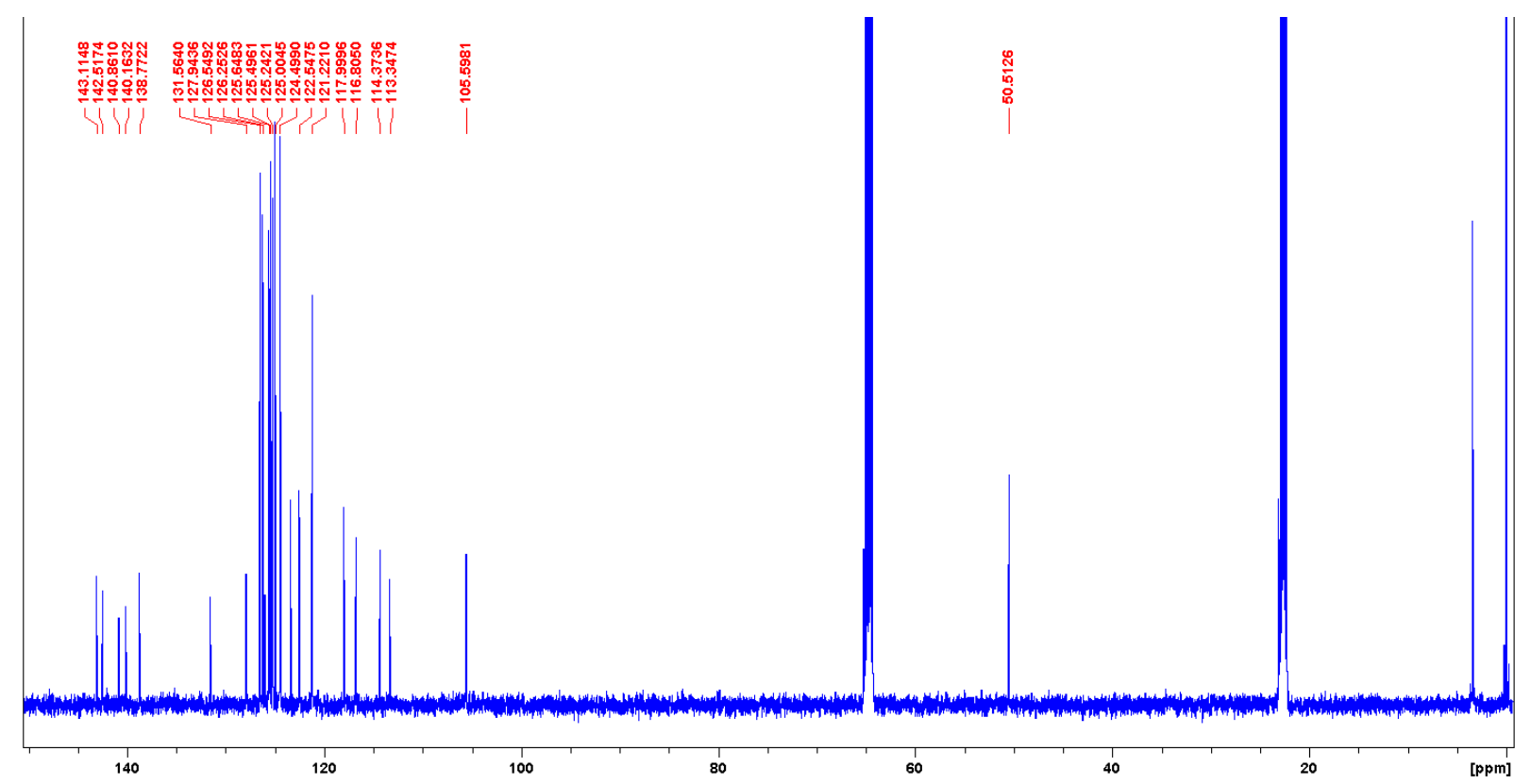

Figure $\mathrm{S} 8:{ }^{13} \mathrm{C}\left\{{ }^{1} \mathrm{H}\right\}-\mathrm{NMR}$ spectrum of $\mathrm{Li}\left[\mathrm{Ph}_{4} \mathrm{PnH}\right]\left(125 \mathrm{MHz}, \mathrm{d}_{8}-\mathrm{THF}, 298 \mathrm{~K}\right)$ 


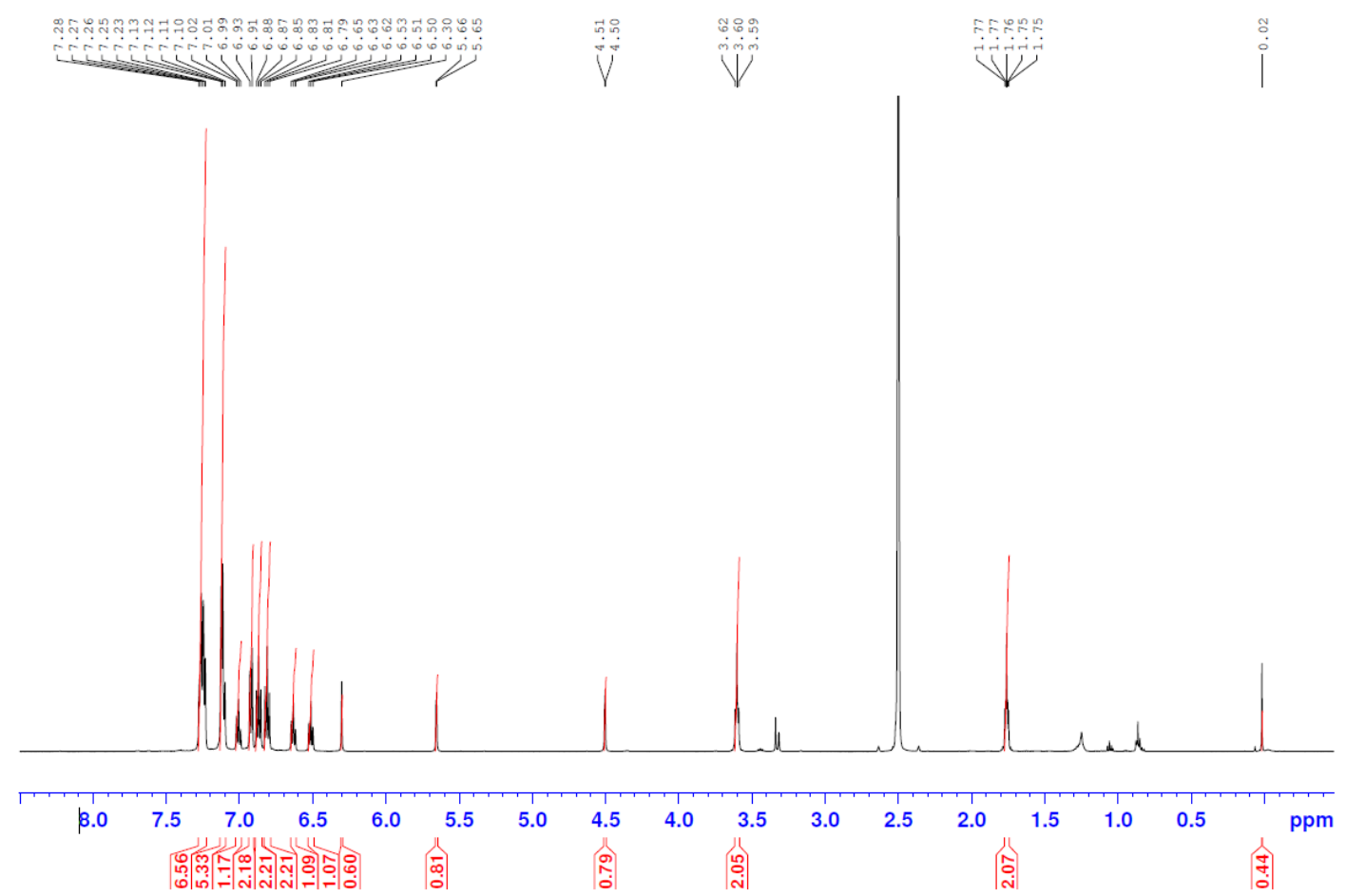

Figure S9: Quantitative ${ }^{1} \mathrm{H}-\mathrm{NMR}$ spectrum of Li(THF) $0_{0.5}\left[\mathrm{Ph}_{4} \mathrm{PnH}\right]\left(500 \mathrm{MHz}, \mathrm{d}_{6}\right.$-DMSO, $\left.298 \mathrm{~K}\right)$

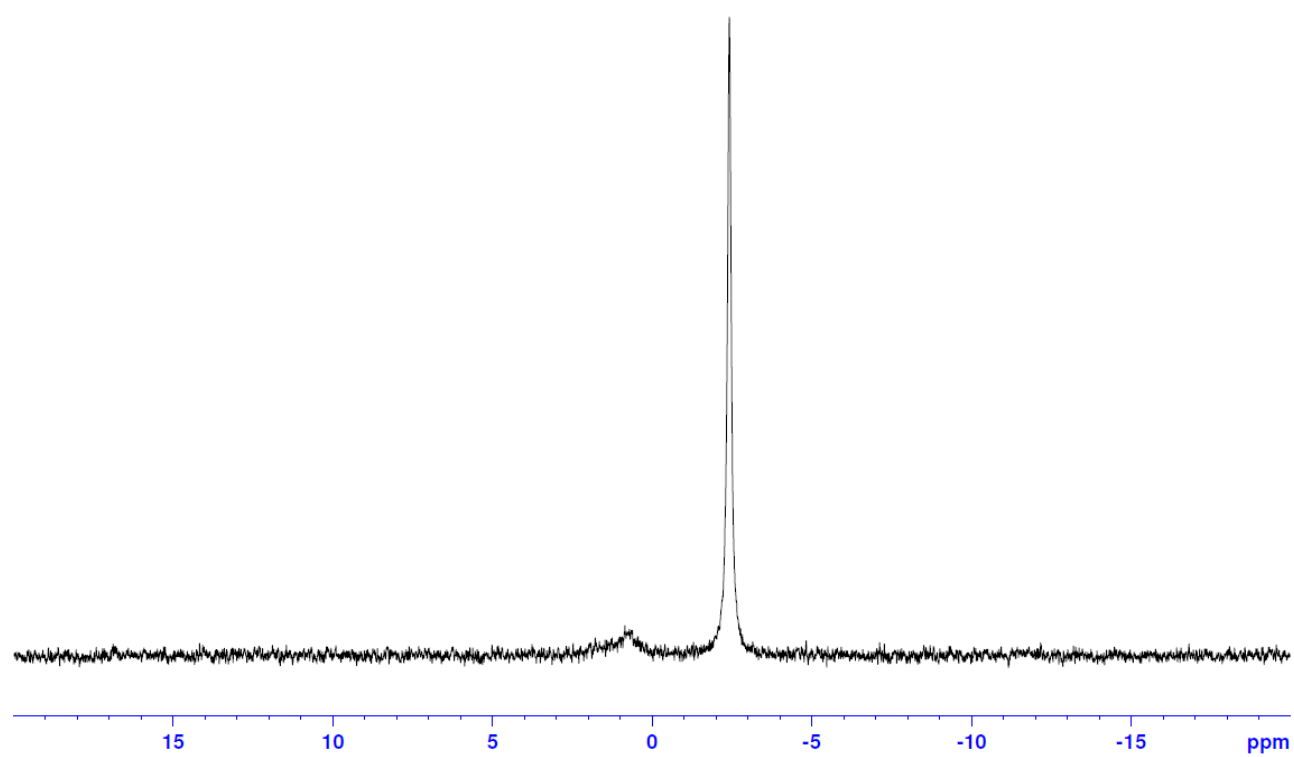

Figure S10: ${ }^{7} \mathrm{Li}-\mathrm{NMR}$ spectrum of Li(THF) $)_{0.5}\left[\mathrm{Ph}_{4} \mathrm{PnH}\right]\left(194 \mathrm{MHz}, \mathrm{h}_{8}\right.$-THF, $\left.298 \mathrm{~K}\right)$ 


\section{d. Sodium 1,3,4,6-tetraphenylhydropentalenide}

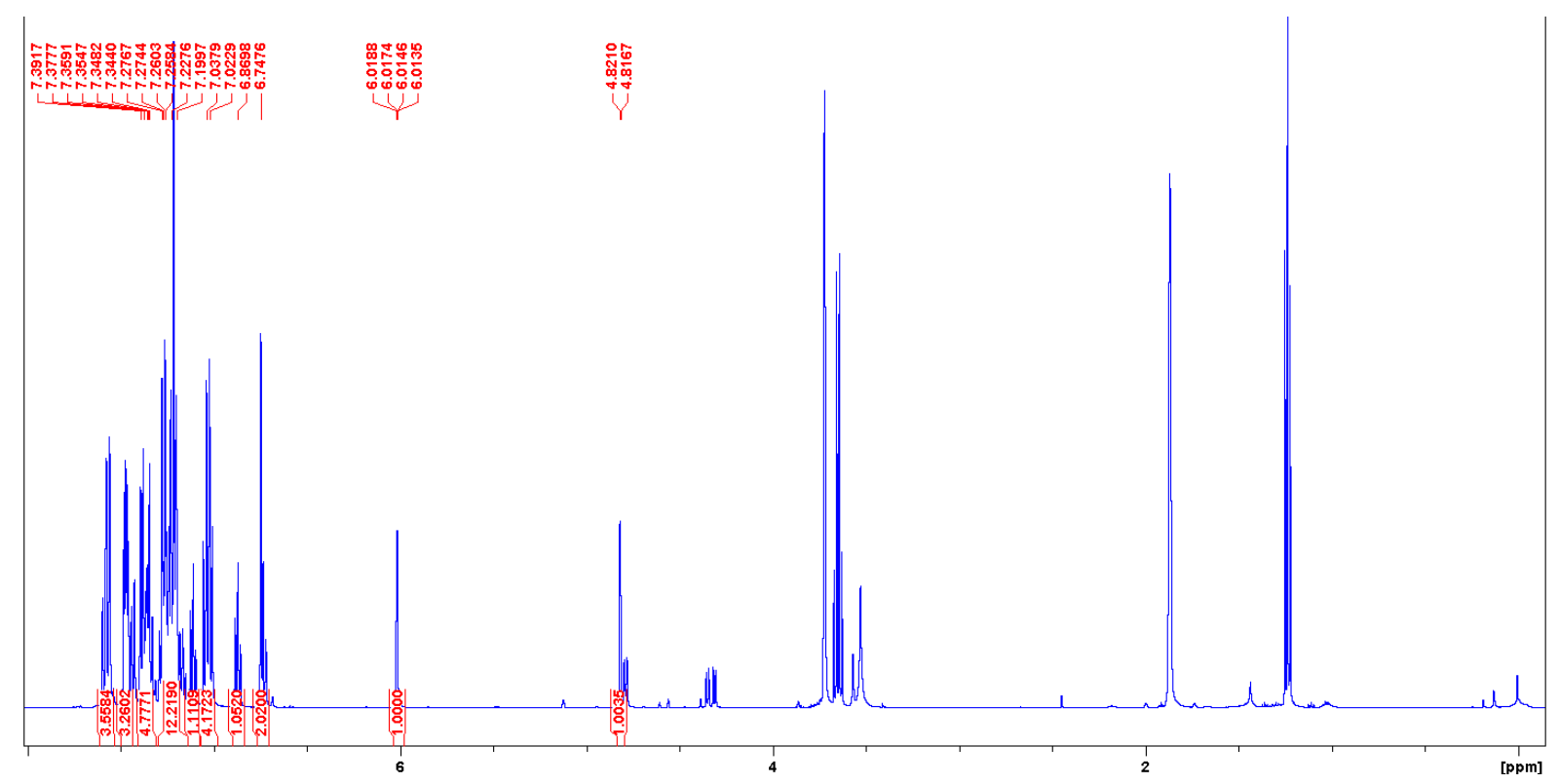

Figure S11: ${ }^{1} \mathrm{H}-\mathrm{NMR}$ spectrum of $\mathrm{Na}\left[\mathrm{Ph}_{4} \mathrm{PnH}\right]\left(500 \mathrm{MHz}, \mathrm{d}_{8}-\mathrm{THF}, 298 \mathrm{~K}\right)$

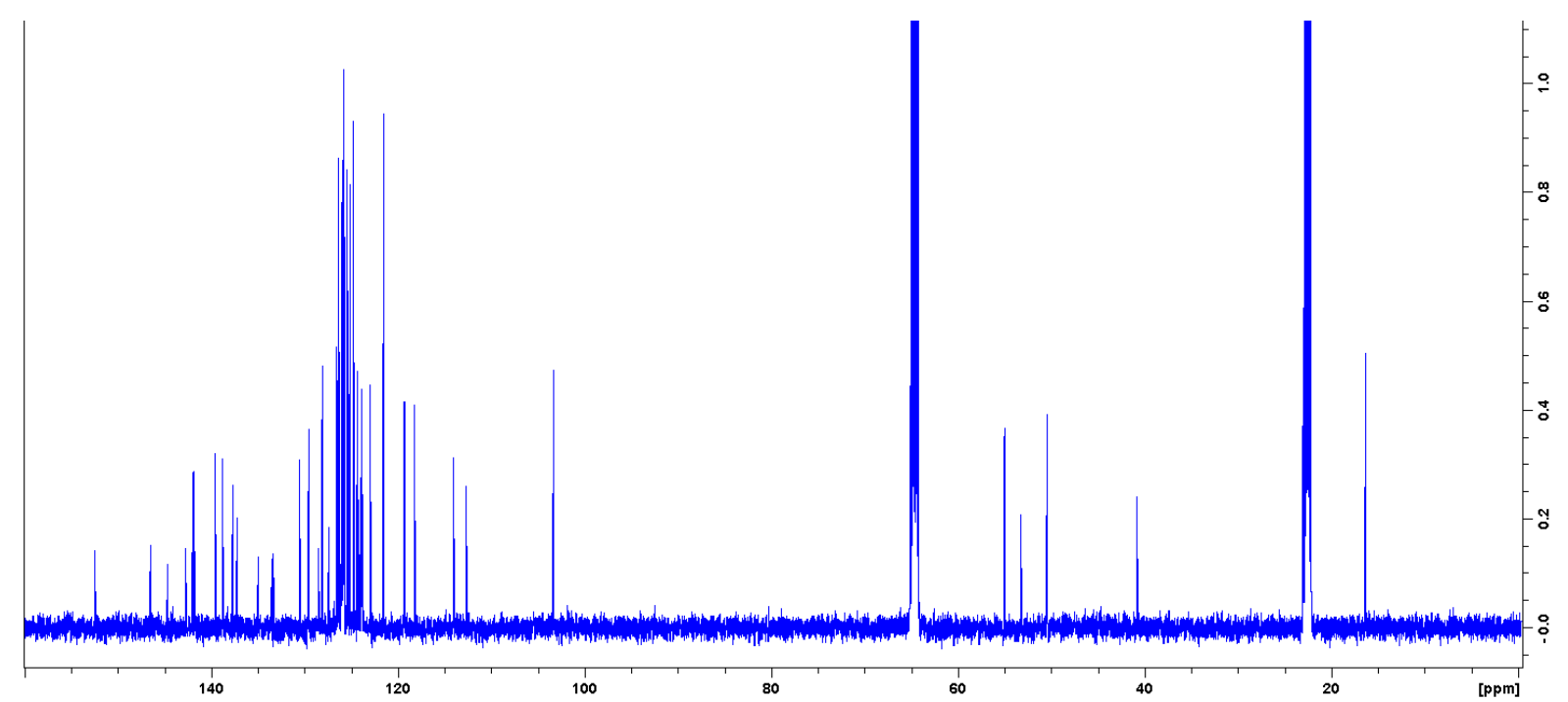

Figure $\mathrm{S} 12:{ }^{13} \mathrm{C}\left\{{ }^{1} \mathrm{H}\right\}$-NMR spectrum of $\mathrm{Na}\left[\mathrm{Ph}_{4} \mathrm{PnH}\right]\left(125 \mathrm{MHz}, \mathrm{d}_{8}-\mathrm{THF}, 298 \mathrm{~K}\right)$ 


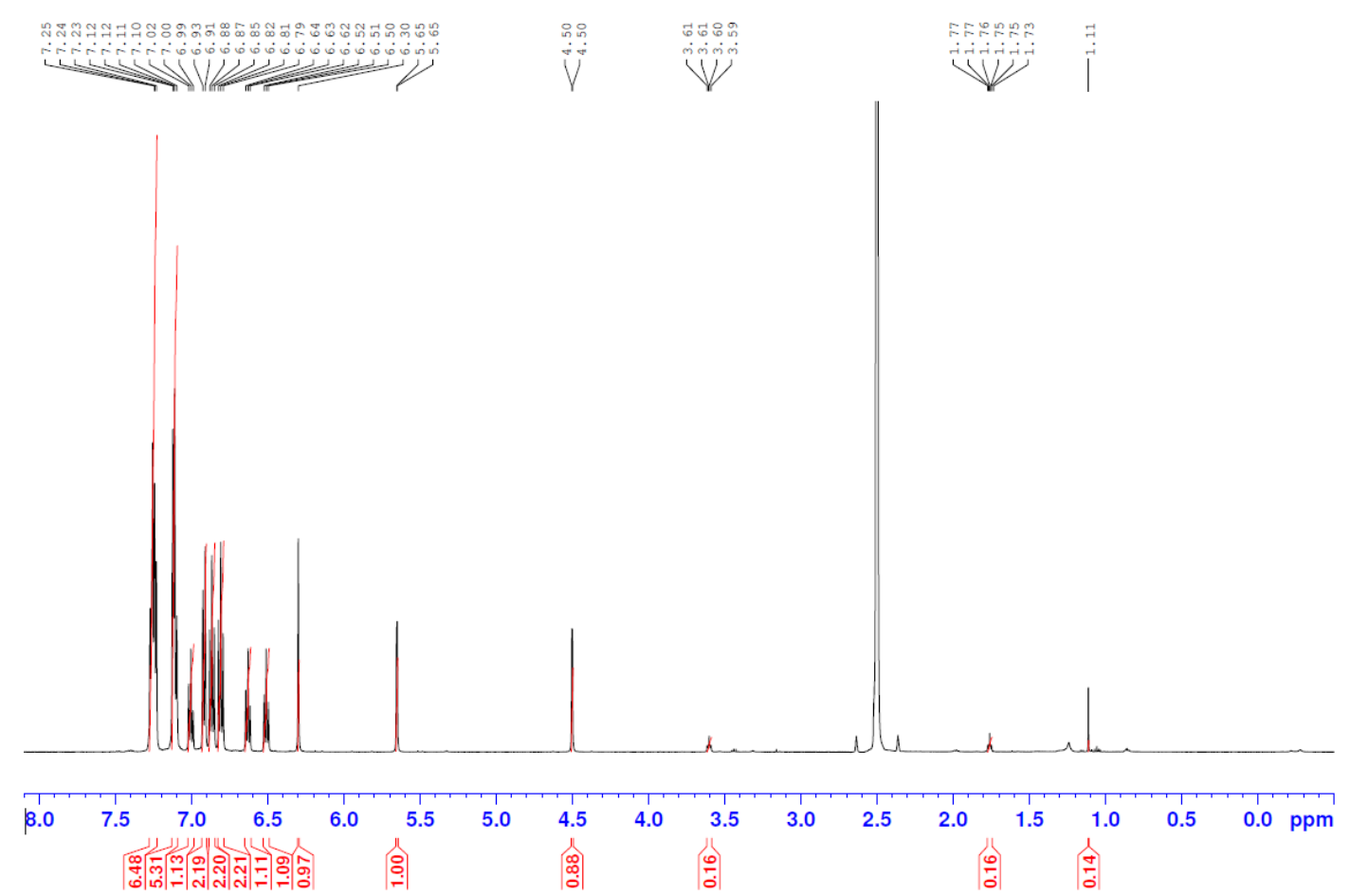

Figure S13: Quantitative ${ }^{1} \mathrm{H}-\mathrm{NMR}$ spectrum of $\mathrm{Na}\left[\mathrm{Ph}_{4} \mathrm{PnH}\right]\left(500 \mathrm{MHz}, \mathrm{d}_{6}-\mathrm{DMSO}, 298 \mathrm{~K}\right.$ )

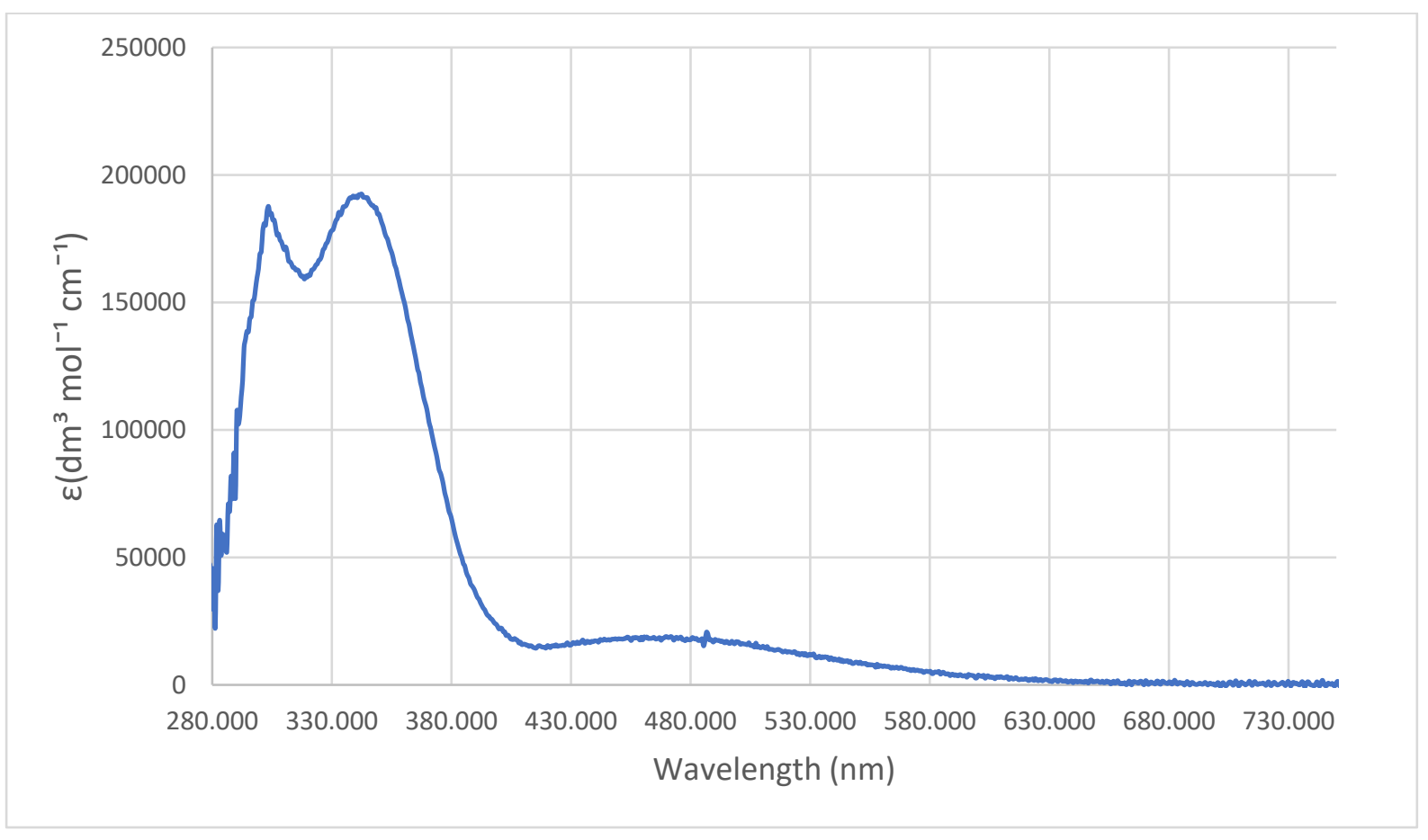

Figure S14: UV/Vis spectrum of $\mathrm{Na}\left[\mathrm{Ph}_{4} \mathrm{PnH}\right]$ in $\mathrm{THF}$ at $293 \mathrm{~K}$ 
e. Potassium 1,3,4,6-tetraphenylhydropentalenide

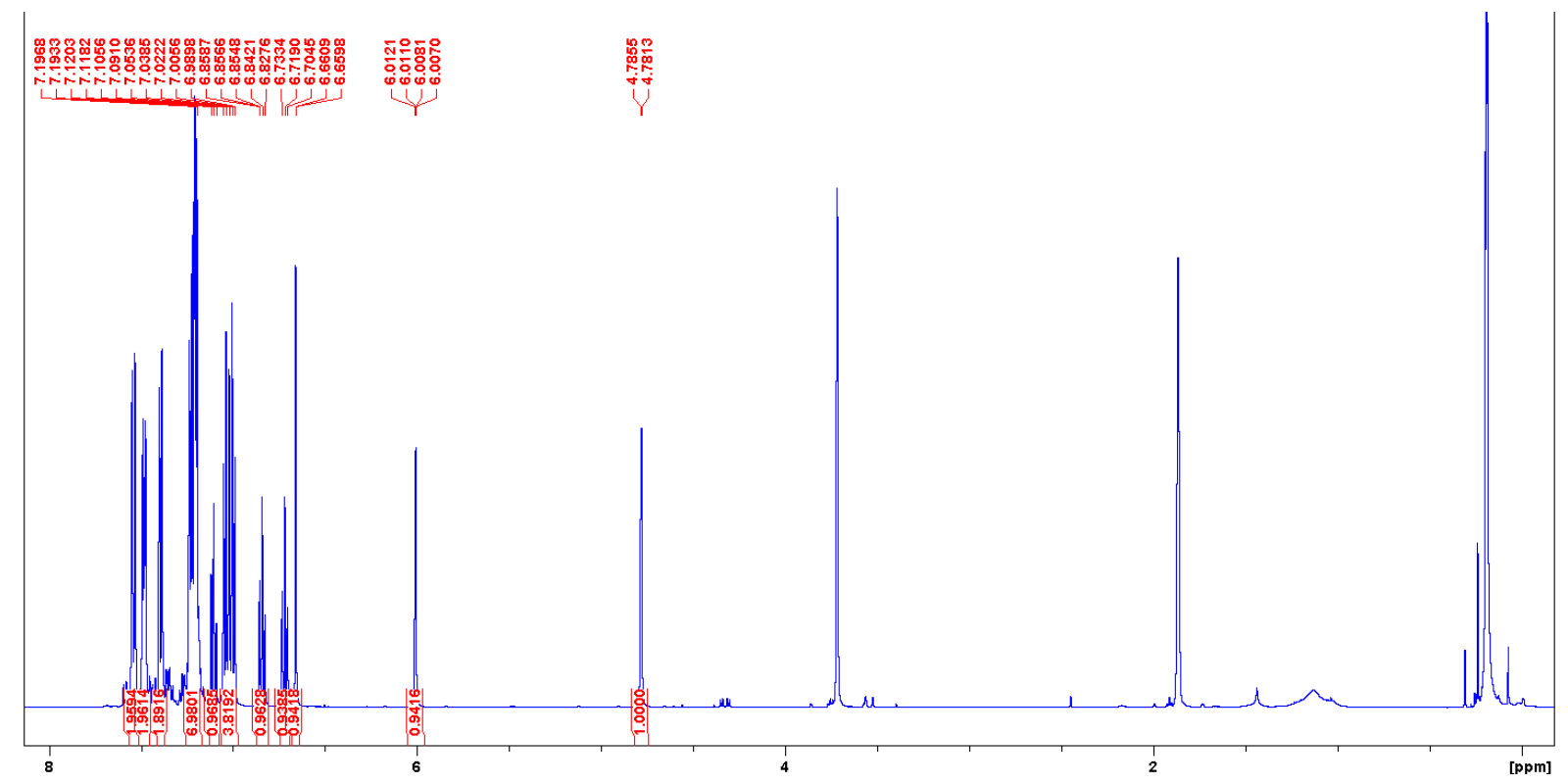

Figure S15: ${ }^{1} \mathrm{H}-\mathrm{NMR}$ spectrum of $\mathrm{K}\left[\mathrm{Ph}_{4} \mathrm{PnH}\right]\left(500 \mathrm{MHz}, \mathrm{d}_{8}-\mathrm{THF}, 298 \mathrm{~K}\right)$

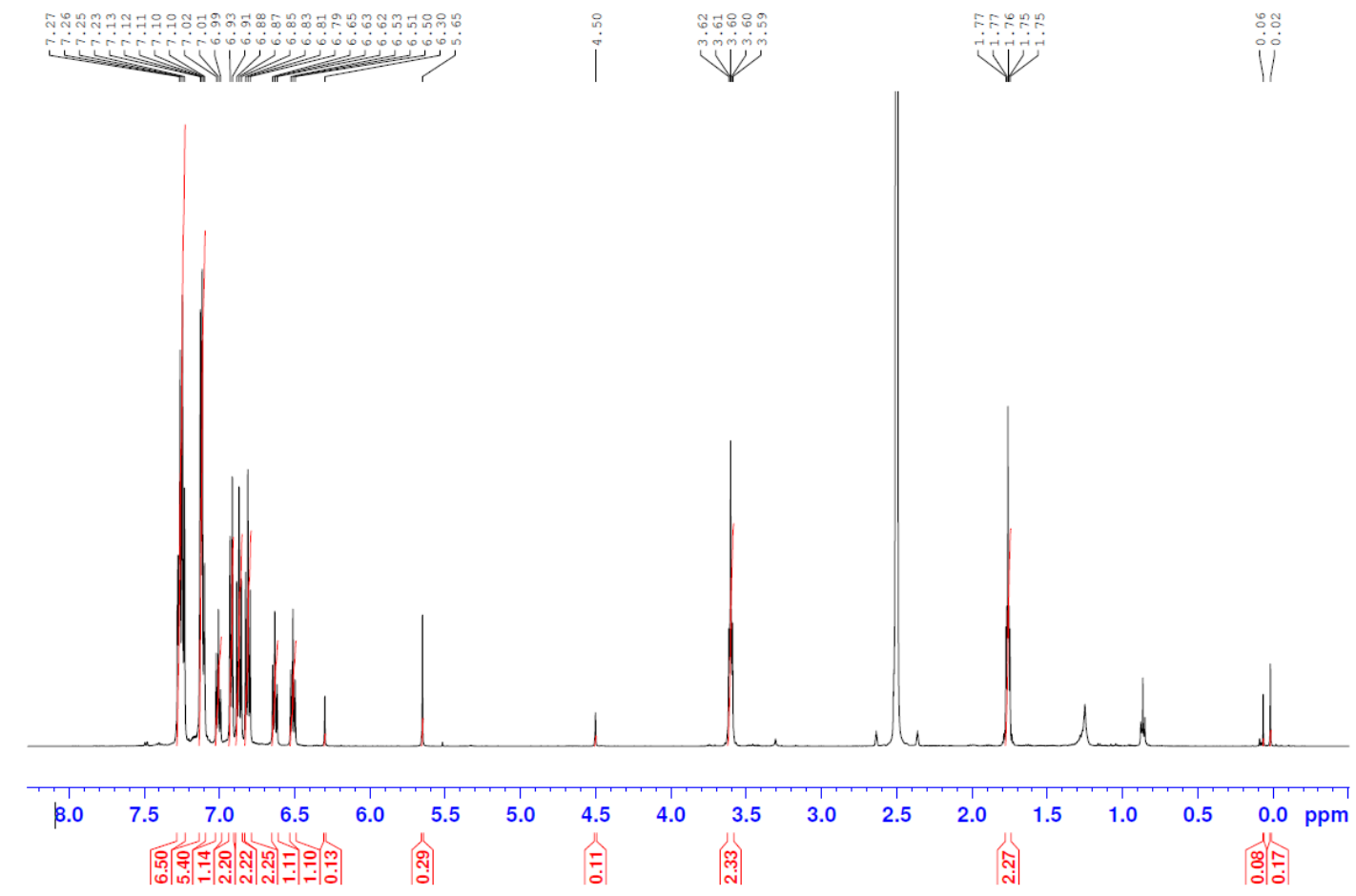

Figure S16: Quantitative ${ }^{1} \mathrm{H}-\mathrm{NMR}$ spectrum of $\mathrm{K}(\mathrm{THF})_{0.6}\left[\mathrm{Ph}_{4} \mathrm{PnH}\right]\left(500 \mathrm{MHz}, \mathrm{d}_{6}-\mathrm{DMSO}, 298 \mathrm{~K}\right)$ 


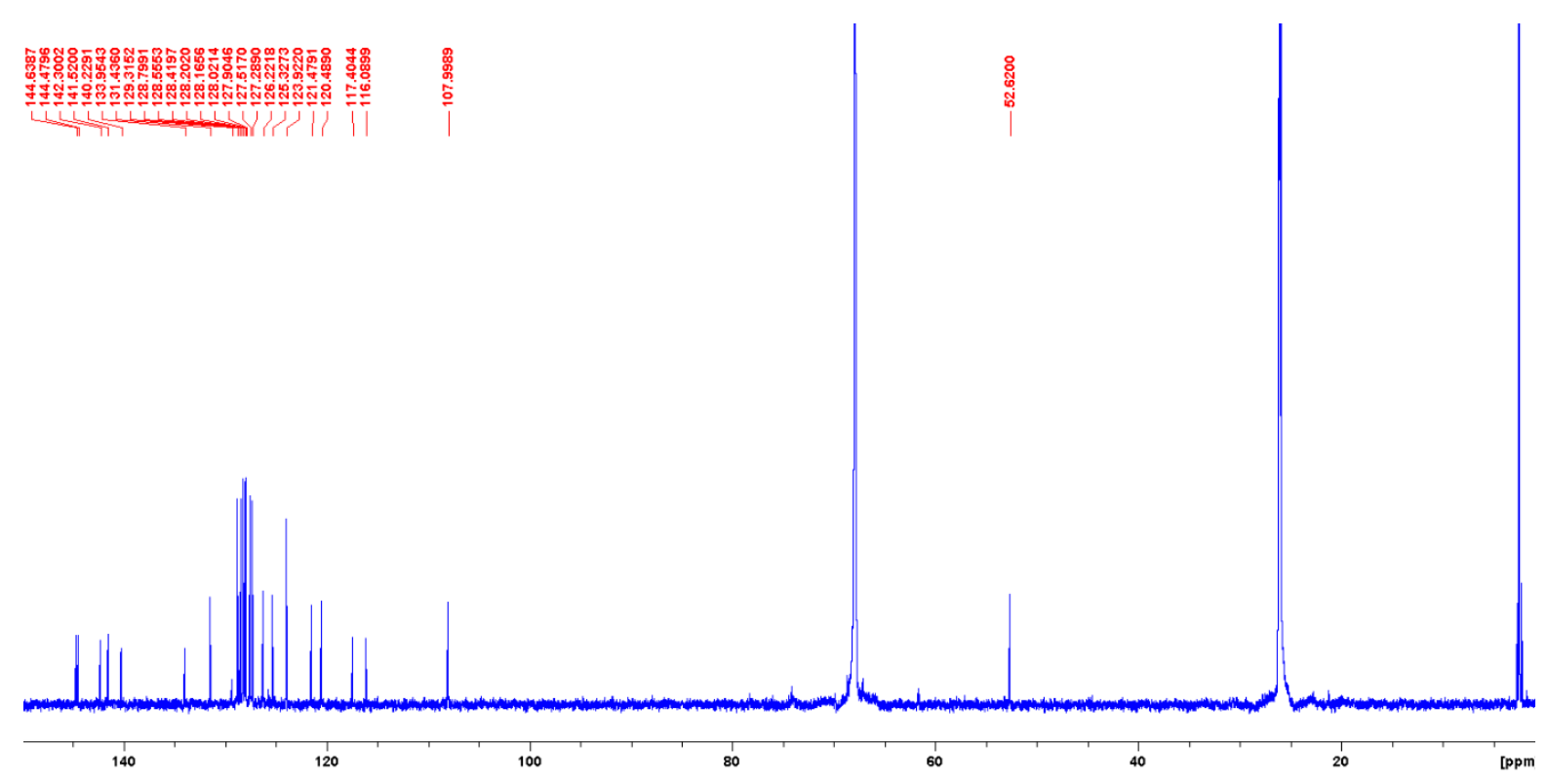

Figure S17: ${ }^{13} \mathrm{C}\left\{{ }^{1} \mathrm{H}\right\}-\mathrm{NMR}$ spectrum of $\mathrm{K}\left[\mathrm{Ph}_{4} \mathrm{PnH}\right]\left(125 \mathrm{MHz}, \mathrm{d}_{8}-\mathrm{THF}, 298 \mathrm{~K}\right)$

f. Lithium potassium 1,3,4,6-tetraphenylpentalenide

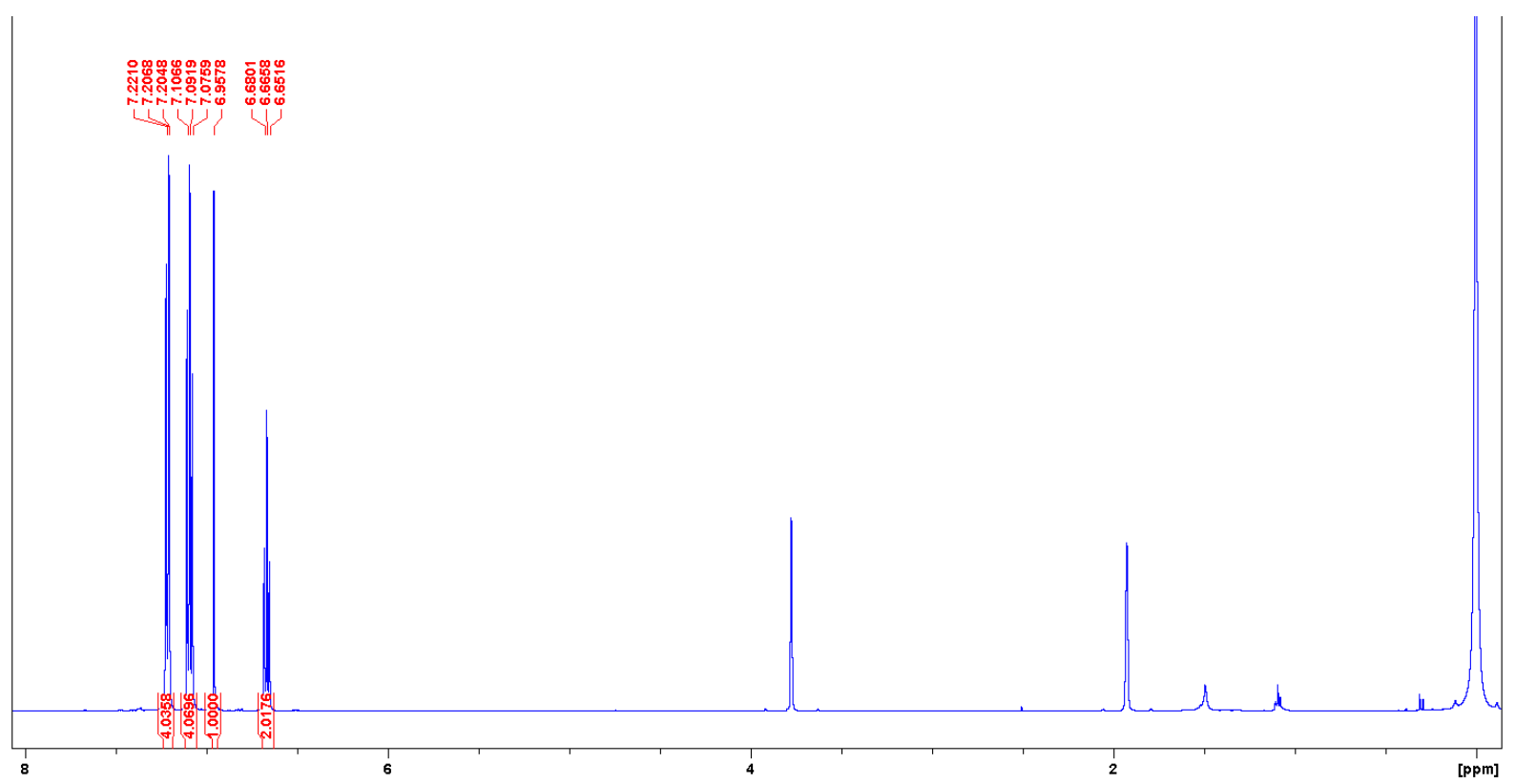

Figure S18: ${ }^{1} \mathrm{H}-\mathrm{NMR}$ spectrum of $\mathrm{LiK}\left[\mathrm{Ph}{ }_{4} \mathrm{Pn}\right]\left(500 \mathrm{MHz}, \mathrm{d}_{8}-\mathrm{THF}, 298 \mathrm{~K}\right)$ 


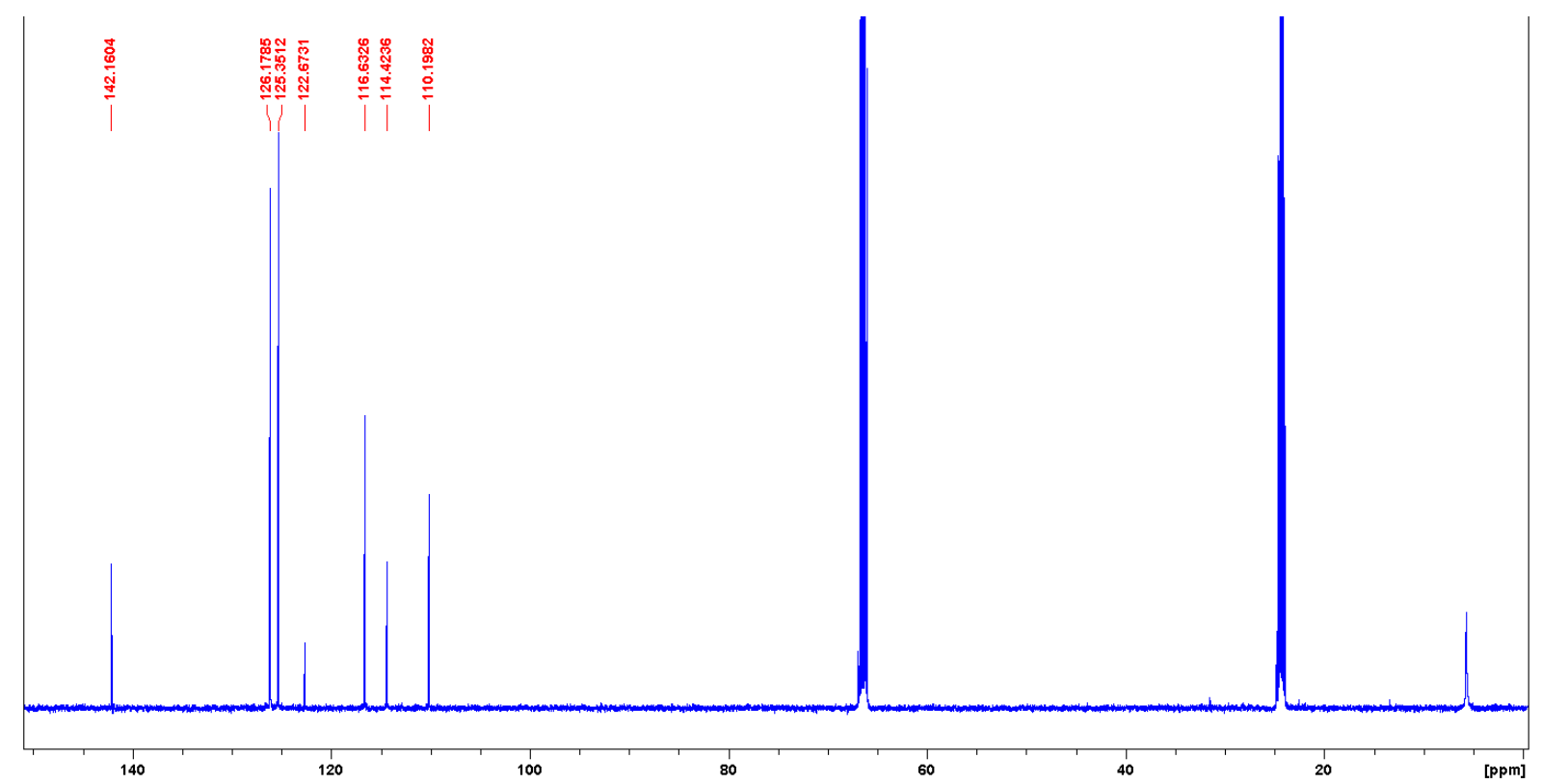

Figure S19: ${ }^{13} \mathrm{C}\left\{{ }^{1} \mathrm{H}\right\}-\mathrm{NMR}$ spectrum of $\mathrm{LiK}\left[\mathrm{Ph}{ }_{4} \mathrm{Pn}\right]\left(125 \mathrm{MHz}, \mathrm{d}_{8}-\mathrm{THF}, 298 \mathrm{~K}\right)$

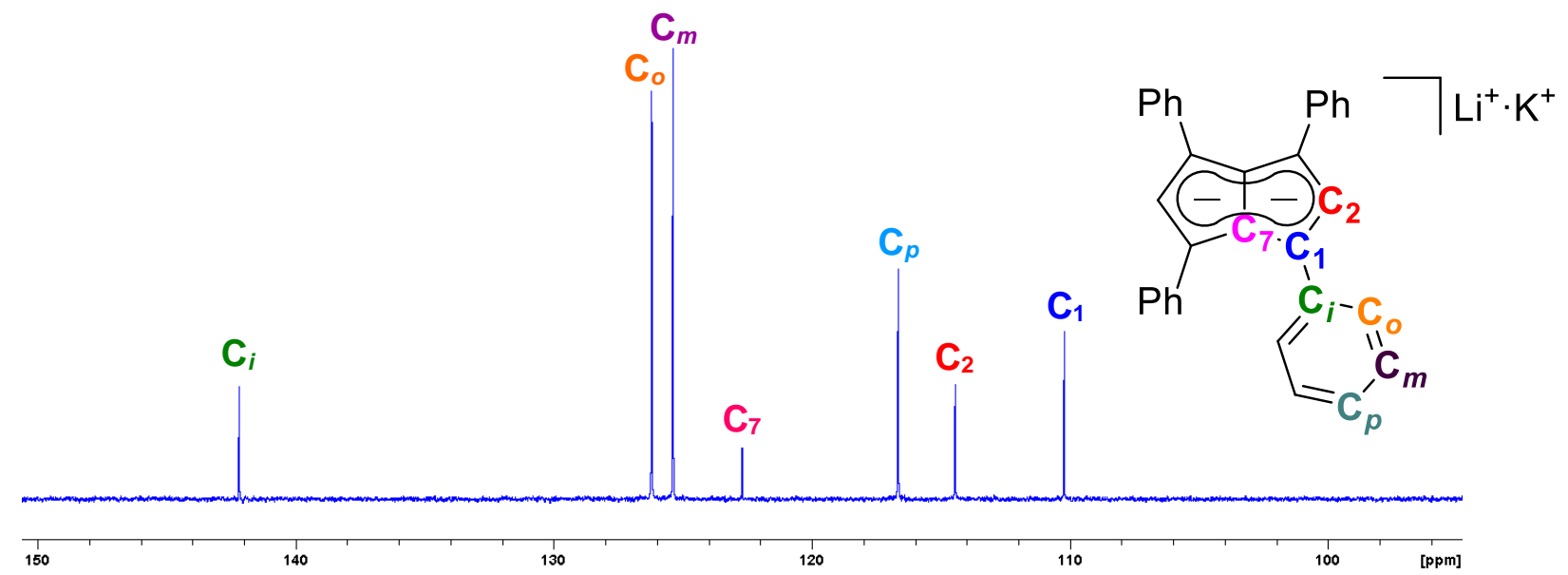

Figure S20: ${ }^{13} \mathrm{C}\{1 \mathrm{H}\}$-NMR spectrum of lithium potassium 1,3,4,6-tetraphenylpentalenide in $\mathrm{d}_{8}$-THF at $25^{\circ} \mathrm{C}$ with carbon assignment 


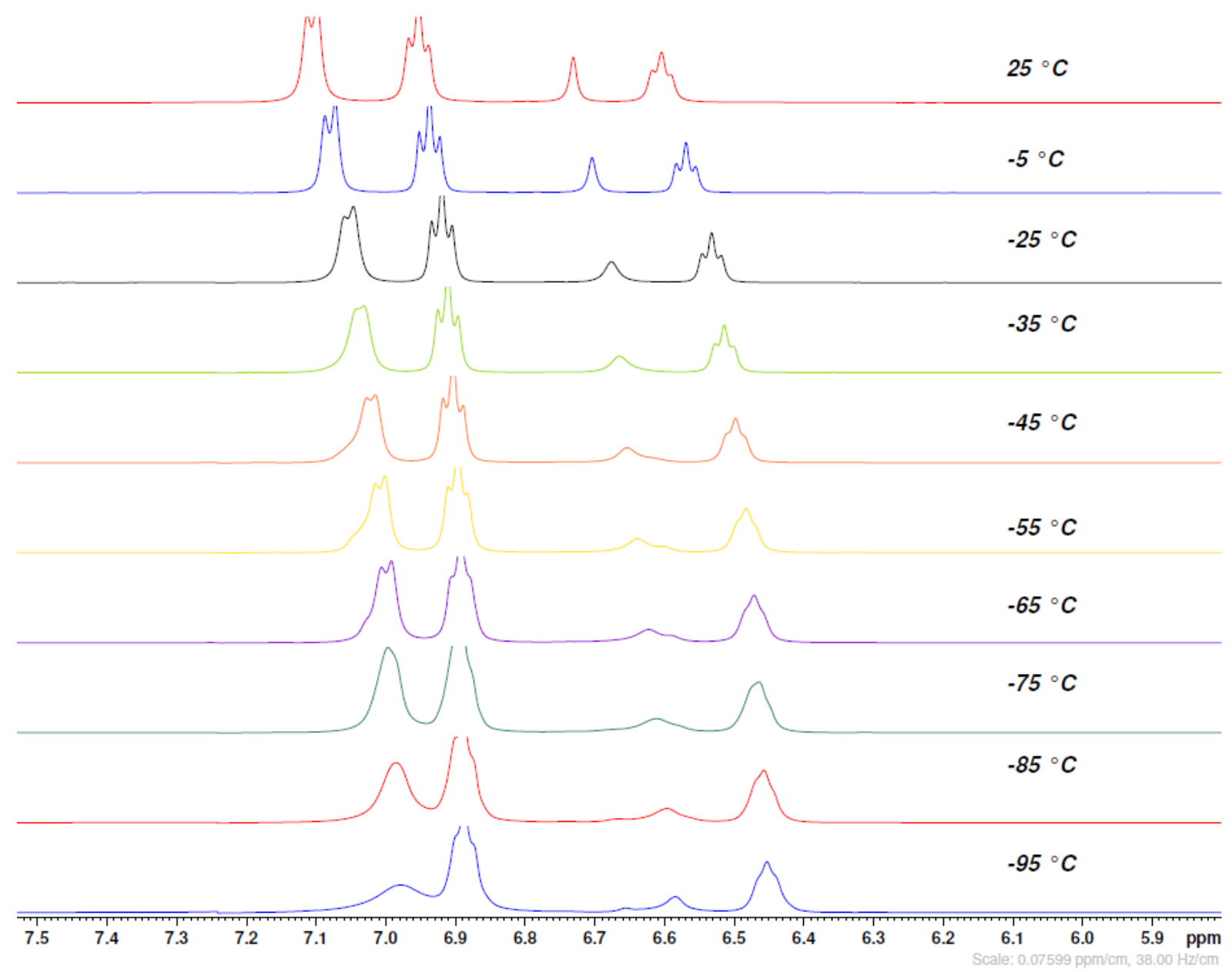

Figure S21: Variable temperature ${ }^{1} \mathrm{H}$-NMR of lithium potassium 1,3,4,6-tetraphenylpentalenide in $\mathrm{h}_{8}$ THF 


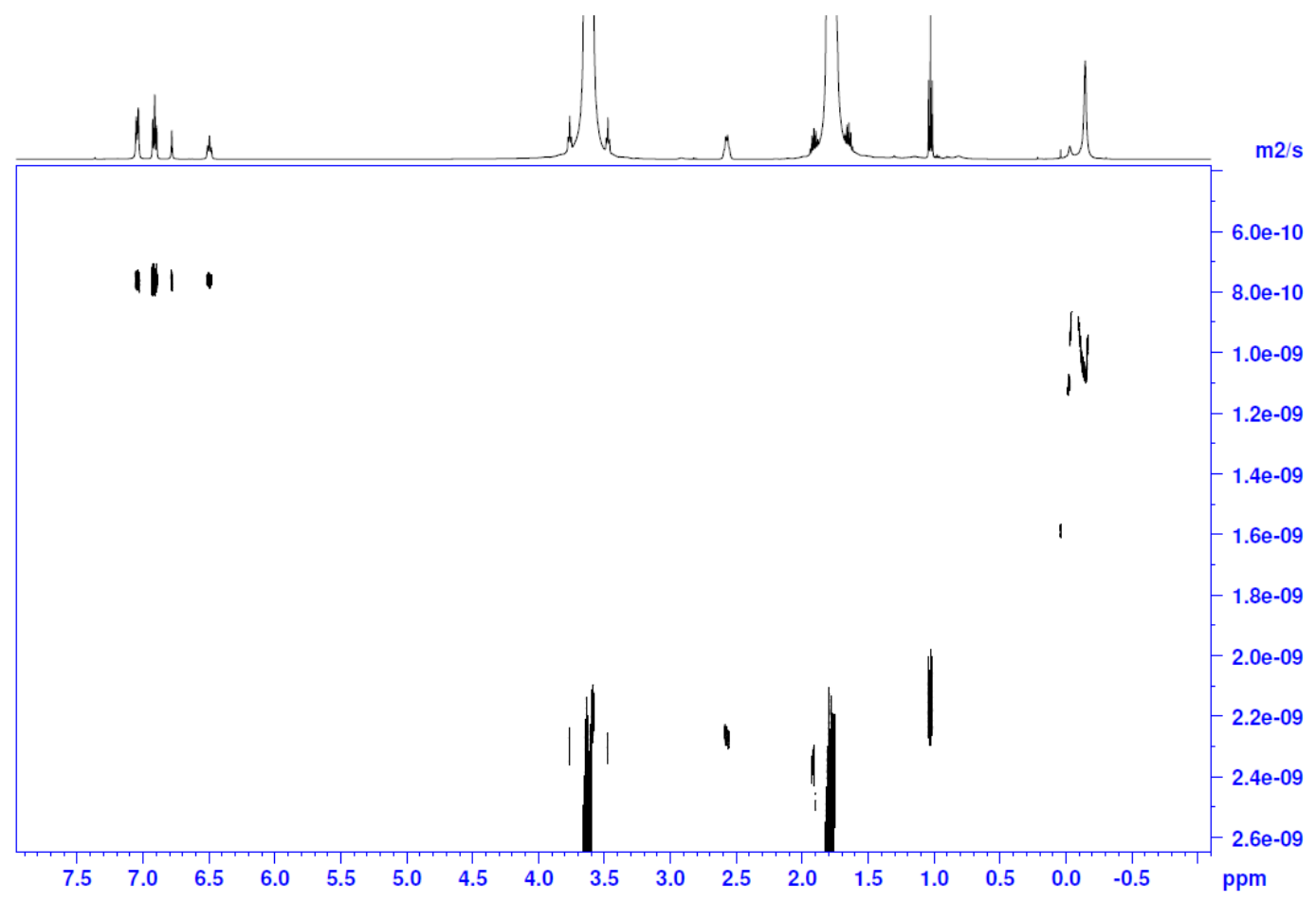

Figure S22: ${ }^{1} \mathrm{H}$ DOSY spectrum of LiK[Ph $\left.{ }_{4} \mathrm{Pn}\right]\left(500 \mathrm{MHz}, \mathrm{h}_{8}-\mathrm{THF}, 298 \mathrm{~K}\right)$ 


\section{g. Sodium potassium 1,3,4,6-tetraphenylpentalenide}

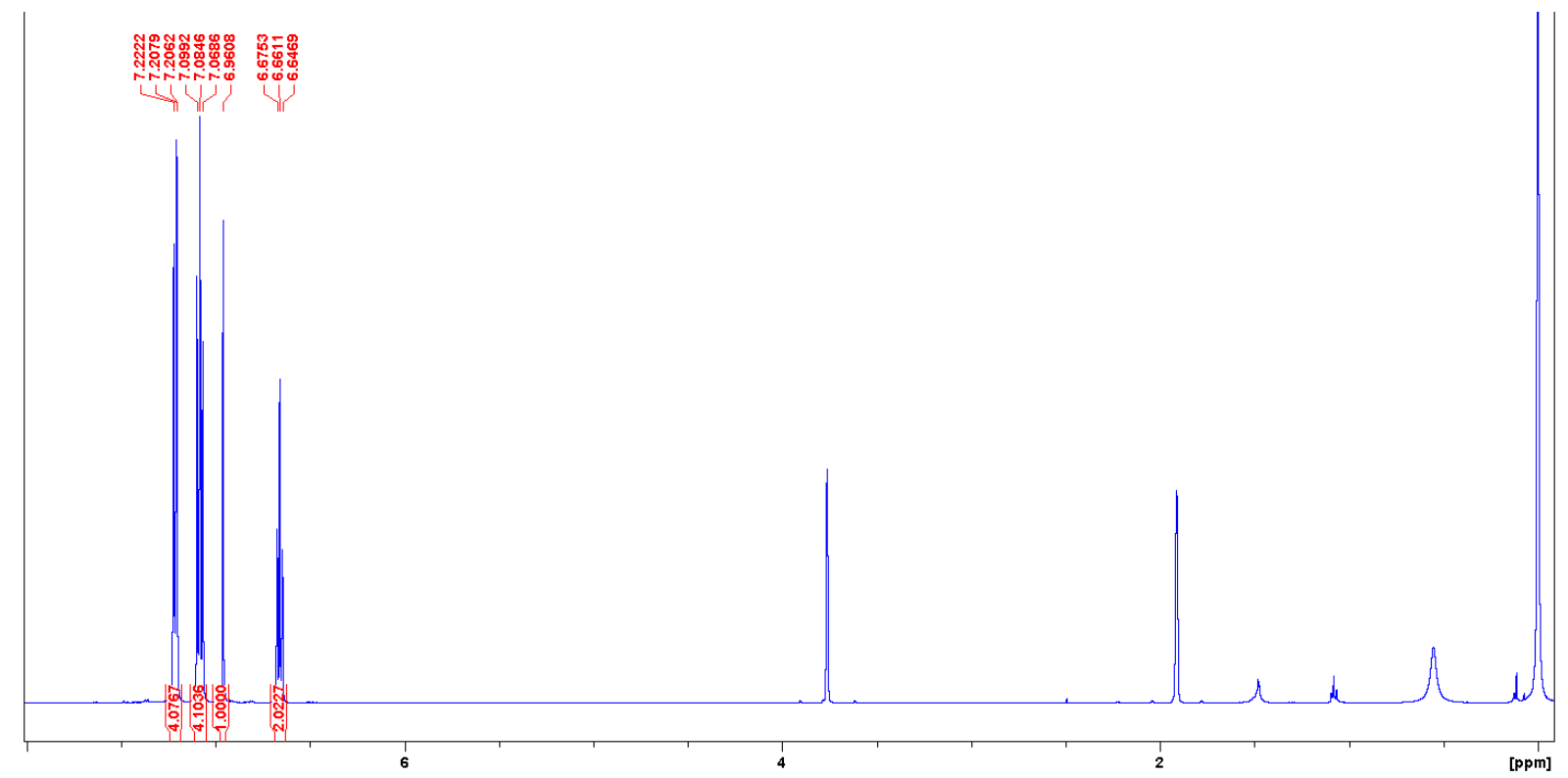

Figure S23: ${ }^{1} \mathrm{H}-\mathrm{NMR}$ spectrum of $\mathrm{NaK}^{\left.\mathrm{P} \mathrm{Ph}_{4} \mathrm{Pn}\right]}\left(500 \mathrm{MHz}, \mathrm{d}_{8}-\mathrm{THF}, 298 \mathrm{~K}\right)$

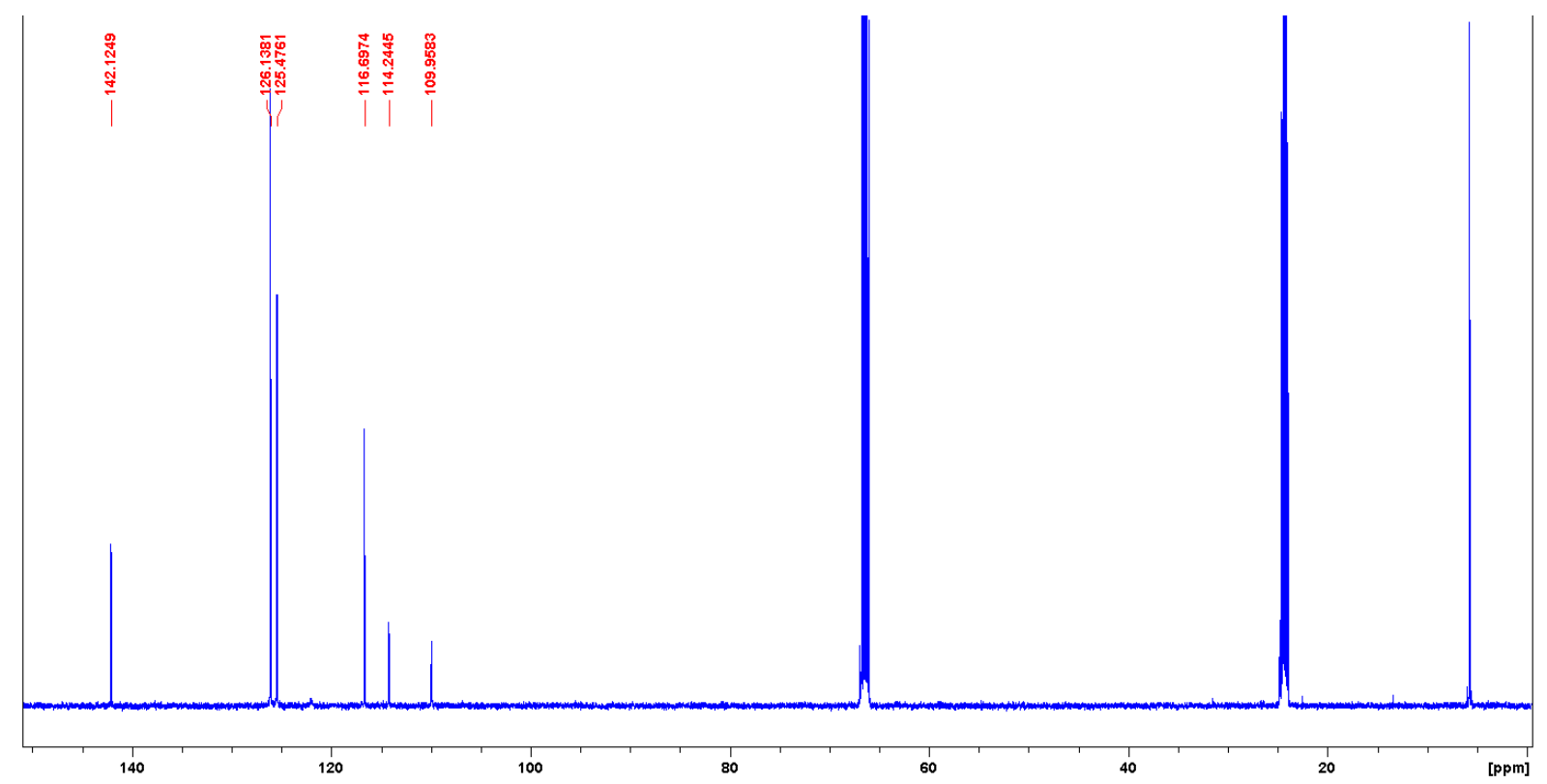

Figure S24: ${ }^{13} \mathrm{C}\left\{{ }^{1} \mathrm{H}\right\}-\mathrm{NMR}$ spectrum of $\mathrm{NaK}\left[\mathrm{Ph}{ }_{4} \mathrm{Pn}\right]\left(125 \mathrm{MHz}, \mathrm{d}_{8}-\mathrm{THF}, 298 \mathrm{~K}\right)$ 


\section{h. Sodium lithium 1,3,4,6-tetraphenylpentalenide}

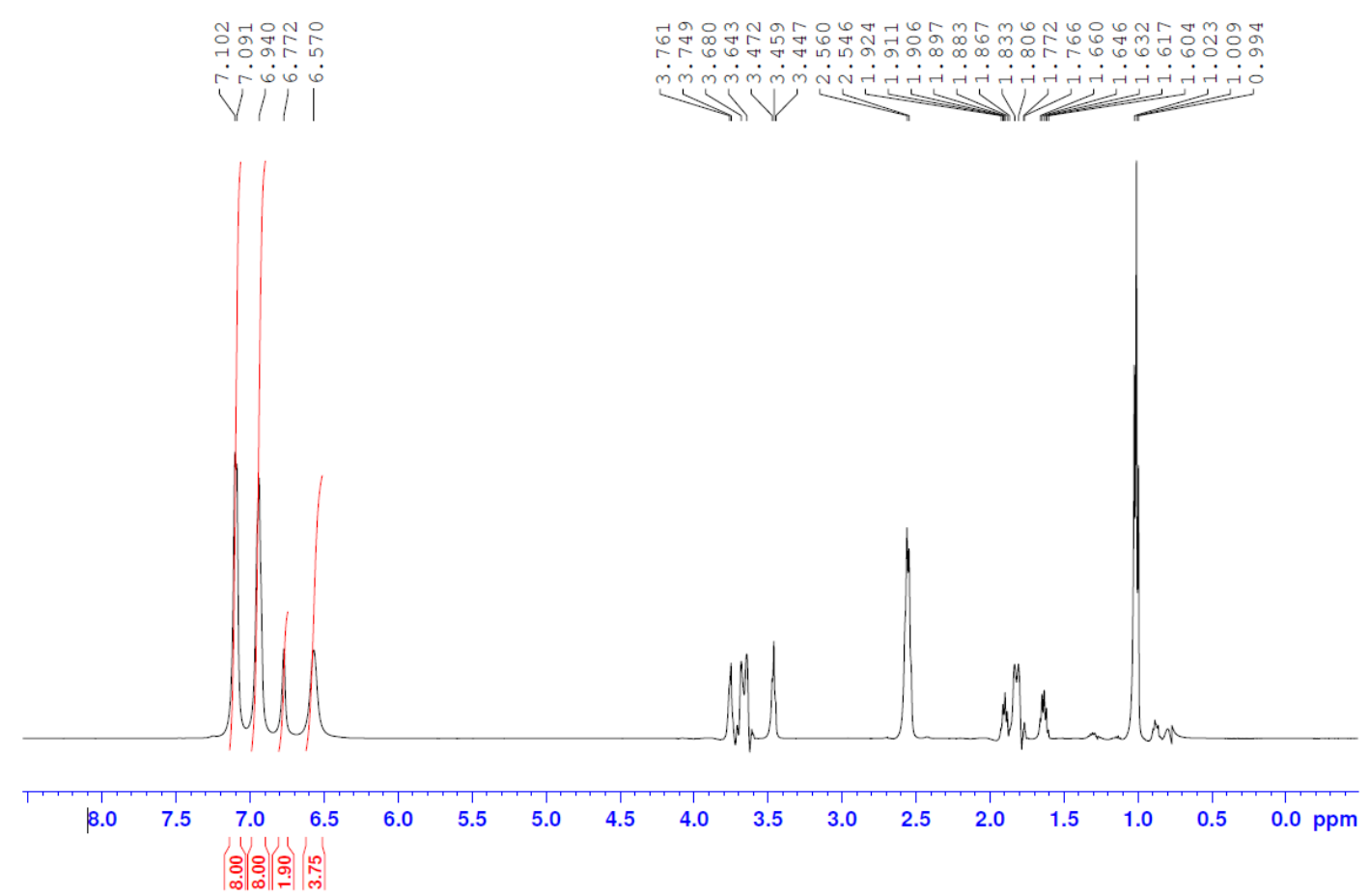

Figure S25: ${ }^{1} \mathrm{H}-\mathrm{NMR}$ spectrum of $\mathrm{NaLi}\left[\mathrm{Ph} \mathrm{P}_{4} \mathrm{Pn}\right]\left(500 \mathrm{MHz}, \mathrm{h}_{8}-\mathrm{THF}, 298 \mathrm{~K}\right)$

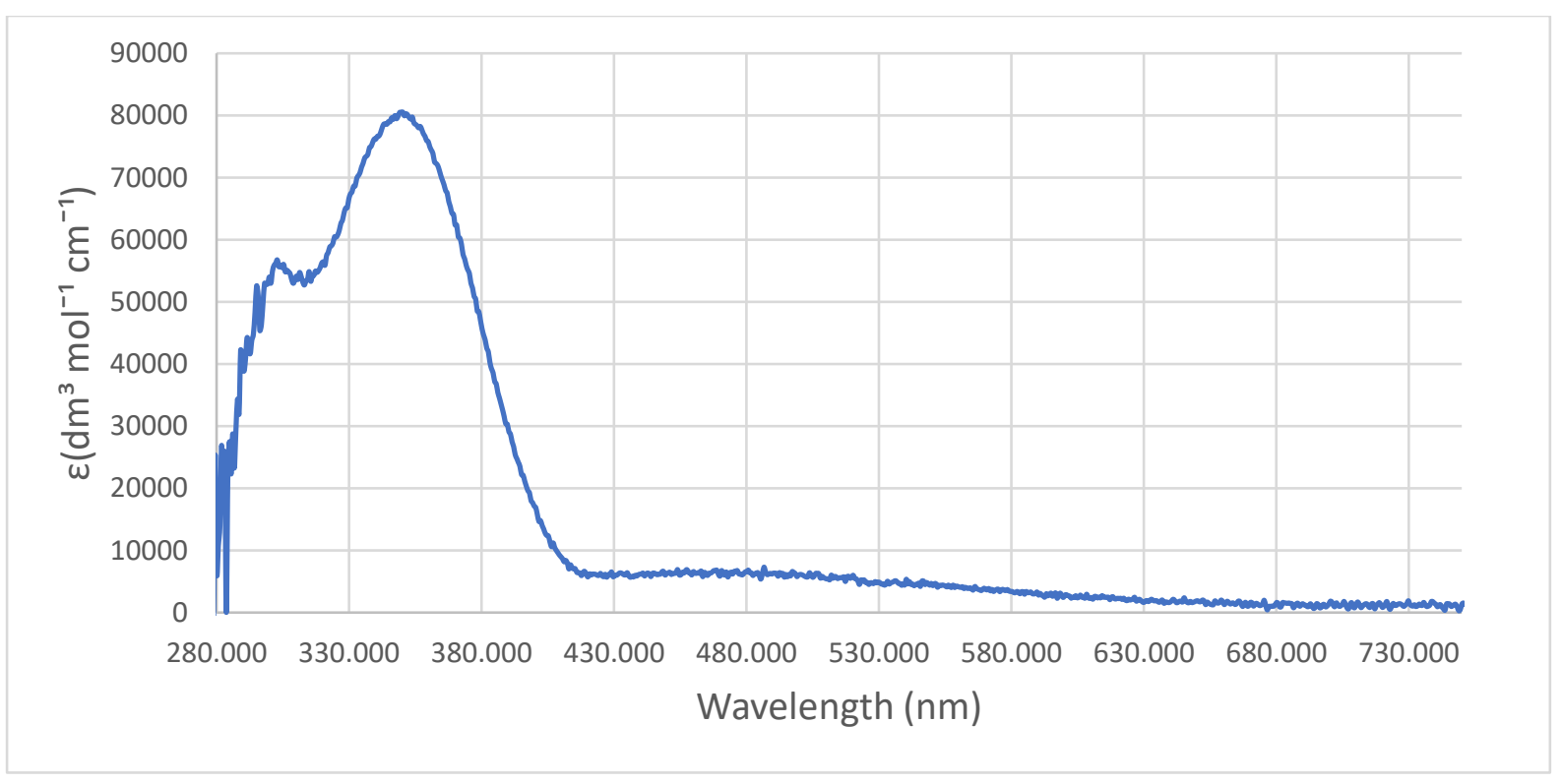

Figure S26: UV/Vis spectrum of $\mathrm{NaLi}\left[\mathrm{Ph}{ }_{4} \mathrm{Pn}\right]$ in THF at $293 \mathrm{~K}$ 


\section{X-ray Crystallography}

Intensity data for e20uh2_sq were collected at 150(2) K on a Rigaku Xcalibur, EosS2 single crystal diffractometer using graphite monochromated Mo-Ka radiation $(\lambda=0.71073 \AA)$ and for s17uh2, s18uh2, s18uh6 and s19uh6 on a Rigaku SuperNova Dual EosS2 single crystal diffractometer using a Cu microfocus source $(\lambda=1.54184 \AA$ A). Unit cell determination, data collection data reduction and absorption correction were performed using the CrysAlisPro software. ${ }^{1}$ The structures were solved with SHELXT ${ }^{2}$ and refined by a full-matrix least-squares procedure based on $F^{2}$ (SHELXL-2018/3). ${ }^{3}$ All non-hydrogen atoms were refined anisotropically. Hydrogen atoms were placed onto calculated positions and refined using a riding model. Data analysis and visualisation was carried out using SHELXIe ${ }^{4}$ and Mercury 5 .

CCDC 2106908-2106913 contain the supplementary crystallographic data for s17uh2, e20uh2, s18uh6, s19uh6, s18uh2 and s19uh7. These data can be obtained free of charge via http://www.ccdc.cam.ac.uk/conts/retrieving.html, or from the Cambridge Crystallographic Data Centre, 12 Union Road, Cambridge CB2 1EZ, UK; fax:(+44) 1223-336-033; or e-mail: deposit@ccdc.cam.ac.uk.

1. CrysAlisPro 1.171.38.46 - 1.171.40.43a (Rigaku Oxford Diffraction, 2015-19).

2. SHELXT: G. M. Sheldrick, ActaCryst, 2015, A71, 3-8,

3. SHELXL: G. M. Sheldrick, ActaCryst, 2015, C71, 3-8.

4. ShelXIe: a Qt graphical user interface for SHELXL: C. B. Hübschle, G. M. Sheldrick and B. Dittrich, J. Appl. Cryst., 44, (2011) 1281-1284.

5. Mercury: C. F. Macrae, P. R. Edgington, P. McCabe, E. Pidcock, G. P. Shields, R. Taylor, Towler and van der Streek, J. Appl. Crystallogr., 2006, 39, 453-457. 


\section{a. Crystal data and structure refinement for $1,3,4,6-$ tetraphenyldihydropentalene}

CCDC number
Identification code
Empirical formula
Formula weight
Temperature
Wavelength
Crystal system
Space group
Unit cell dimensions

Volume

Z

Density (calculated)

Absorption coefficient

$\mathrm{F}(000)$

Crystal size

Theta range for data collection

Index ranges

Reflections collected

Independent reflections

Completeness to theta $=67.684^{\circ}$

Absorption correction

Max. and min. transmission

Refinement method

Data / restraints / parameters

Goodness-of-fit on $\mathrm{F}^{2}$

Final $R$ indices [ $\mid>2 \operatorname{sigma}(I)]$

$\mathrm{R}$ indices (all data)

Extinction coefficient

Largest diff. peak and hole
2106908

s17uh2

$\mathrm{C}_{32} \mathrm{H}_{24}$

408.51

150.00(10) K

$1.54184 \AA$

Monoclinic

12/a

$$
\begin{array}{ll}
a=22.4778(7) \AA & \alpha=90^{\circ} \\
b=5.7087(2) \AA & \beta=91.370(3)^{\circ} \\
c=17.2058(5) \AA & \gamma=90^{\circ}
\end{array}
$$

2207.20(12) $\AA^{3}$

4

$1.229 \mathrm{Mg} / \mathrm{m}^{3}$

$0.524 \mathrm{~mm}^{-1}$

864

$0.200 \times 0.150 \times 0.040 \mathrm{~mm}^{3}$

3.934 to $73.020^{\circ}$

$-26<=h<=27,-5<=k<=7,-21<=1<=21$

6989

$2188[R$ (int) $=0.0230]$

$100.0 \%$

Semi-empirical from equivalents

1.00000 and 0.80138

Full-matrix least-squares on $\mathrm{F}^{2}$

$2188 / 0 / 235$

1.222

$\mathrm{R} 1=0.0735, \mathrm{wR} 2=0.1552$

$R 1=0.0790, w R 2=0.1574$

$\mathrm{n} / \mathrm{a}$

0.147 and -0.182 e. $\AA^{-3}$ 


\section{b. Crystal data and structure refinement for potassium 1,3,4,6- tetraphenylhydropentalenide}

CCDC number
Identification code
Empirical formula
Formula weight
Temperature
Wavelength
Crystal system
Space group
Unit cell dimensions

Volume

Z

Density (calculated)

Absorption coefficient

$\mathrm{F}(000)$

Crystal size

Theta range for data collection

Index ranges

Reflections collected

Independent reflections

Completeness to theta $=25.237^{\circ}$

Absorption correction

Max. and min. transmission

Refinement method

Data / restraints / parameters

Goodness-of-fit on $\mathrm{F}^{2}$

Final $R$ indices [ $\mid>2 \operatorname{sigma}(I)]$

$\mathrm{R}$ indices (all data)

Extinction coefficient

Largest diff. peak and hole
2106909

e20uh2_sq

$\mathrm{C}_{76} \mathrm{H}_{70} \mathrm{~K}_{2} \mathrm{O}_{3}$

1109.52

149.99(19) K

$0.71073 \AA$

Monoclinic

$\mathrm{P} 22_{1} / \mathrm{c}$

$\mathrm{a}=12.9907(7) \AA$

$\alpha=90^{\circ}$

$\mathrm{b}=30.3440(12) \AA$

$\beta=109.934(6)^{\circ}$

$\mathrm{c}=18.5874(10) \AA$

$\gamma=90^{\circ}$

$6888.0(6) \AA^{3}$

4

$1.070 \mathrm{Mg} / \mathrm{m}^{3}$

$0.181 \mathrm{~mm}^{-1}$

2352

$0.597 \times 0.091 \times 0.064 \mathrm{~mm}^{3}$

2.927 to $25.237^{\circ}$

$-15<=h<=15,-36<=k<=36,-22<=\mid<=22$

97309

$12398[R($ int $)=0.1513]$

$99.4 \%$

Semi-empirical from equivalents

1.00000 and 0.91985

Full-matrix least-squares on $\mathrm{F}^{2}$

12398 / 81 / 760

0.990

$\mathrm{R} 1=0.0698, \mathrm{wR} 2=0.1449$

$R 1=0.1514, w R 2=0.1767$

$\mathrm{n} / \mathrm{a}$

0.838 and -0.638 e. $\AA^{-3}$ 


\section{c. Crystal data and structure refinement for dilithium 1,3,4,6- tetraphenylpentalenide}

CCDC number
Identification code
Empirical formula
Formula weight
Temperature
Wavelength
Crystal system
Space group
Unit cell dimensions

Volume

Z

Density (calculated)

Absorption coefficient

$\mathrm{F}(000)$

Crystal size

Theta range for data collection

Index ranges

Reflections collected

Independent reflections

Completeness to theta $=67.684^{\circ}$

Absorption correction

Max. and min. transmission

Refinement method

Data / restraints / parameters

Goodness-of-fit on $\mathrm{F}^{2}$

Final R indices [ $1>2 \operatorname{sigma}(I)]$

$\mathrm{R}$ indices (all data)

Extinction coefficient

Largest diff. peak and hole
2106910

s18uh6

$\mathrm{C}_{48} \mathrm{H}_{54} \mathrm{Li}_{2} \mathrm{O}_{4}$

708.79

150.00(10) K

$1.54184 \AA$

Monoclinic

$\mathrm{P} 21 / \mathrm{n}$

$$
\begin{array}{ll}
a=14.2476(2) \AA & \alpha=90^{\circ} \\
b=8.82290(10) \AA & \beta=93.4200(10)^{\circ} \\
c=16.0040(3) \AA & \gamma=90^{\circ}
\end{array}
$$

2008.20(5) $\AA^{3}$

2

$1.172 \mathrm{Mg} / \mathrm{m}^{3}$

$0.556 \mathrm{~mm}^{-1}$

760

$0.514 \times 0.411 \times 0.152 \mathrm{~mm}^{3}$

4.284 to $73.162^{\circ}$

$-17<=\mathrm{h}<=17,-8<=\mathrm{k}<=10,-19<=\mid<=19$

22952

$4008[R($ int $)=0.0396]$

$100.0 \%$

Semi-empirical from equivalents

1.00000 and 0.38597

Full-matrix least-squares on $\mathrm{F}^{2}$

4008 / 0 / 262

1.039

$R 1=0.0506, w R 2=0.1340$

$\mathrm{R} 1=0.0544, \mathrm{wR} 2=0.1380$

$\mathrm{n} / \mathrm{a}$

0.388 and -0.360 e. $\AA^{-3}$ 


\section{d. Crystal data and structure refinement for disodium 1,3,4,6- tetraphenylpentalenide}

CCDC number
Identification code
Empirical formula
Formula weight
Temperature
Wavelength
Crystal system
Space group
Unit cell dimensions

Volume

Z

Density (calculated)

Absorption coefficient

$\mathrm{F}(000)$

Crystal size

Theta range for data collection

Index ranges

Reflections collected

Independent reflections

Completeness to theta $=67.684^{\circ}$

Absorption correction

Max. and min. transmission

Refinement method

Data / restraints / parameters

Goodness-of-fit on $\mathrm{F}^{2}$

Final $\mathrm{R}$ indices [ $\mid>2 \operatorname{sigma}(\mathrm{I})]$

$R$ indices (all data)

Extinction coefficient

Largest diff. peak and hole
2106911

s19uh6

$\mathrm{C}_{56} \mathrm{H}_{70} \mathrm{Na}_{2} \mathrm{O}_{6}$

885.10

150.01(10) K

$1.54184 \AA$

Monoclinic

$\mathrm{P} 22_{1} / \mathrm{n}$

$a=10.75710(10) \AA$

$\alpha=90^{\circ}$

$\mathrm{b}=12.92160(10) \AA$

$\beta=97.8400(10)^{\circ}$

$c=17.4019(2) \AA$

$\gamma=90^{\circ}$

2396.24(4) $\AA^{3}$

2

$1.227 \mathrm{Mg} / \mathrm{m}^{3}$

$0.767 \mathrm{~mm}^{-1}$

952

$0.290 \times 0.202 \times 0.141 \mathrm{~mm}^{3}$

4.276 to $73.115^{\circ}$

$-13<=\mathrm{h}<=13,-16<=\mathrm{k}<=16,-21<=\mid<=21$

46889

$4790[R($ int $)=0.0333]$

$100.0 \%$

Semi-empirical from equivalents

1.00000 and 0.82349

Full-matrix least-squares on $\mathrm{F}^{2}$

4790 / 0 / 298

1.019

$\mathrm{R} 1=0.0381, \mathrm{wR} 2=0.0964$

$R 1=0.0406, w R 2=0.0983$

$\mathrm{n} / \mathrm{a}$

0.316 and -0.326 e. $\AA^{-3}$ 


\section{e. Crystal data and structure refinement for thf-coordinated dipotassium 1,3,4,6-tetraphenylpentalenide}

CCDC number
Identification code
Empirical formula
Formula weight
Temperature
Wavelength
Crystal system
Space group
Unit cell dimensions

Volume

Z

Density (calculated)

Absorption coefficient

$\mathrm{F}(000)$

Crystal size

Theta range for data collection

Index ranges

Reflections collected

Independent reflections

Completeness to theta $=67.684^{\circ}$

Absorption correction

Max. and min. transmission

Refinement method

Data / restraints / parameters

Goodness-of-fit on $\mathrm{F}^{2}$

Final $R$ indices [ $\mid>2 \operatorname{sigma}(I)]$

$R$ indices (all data)

Extinction coefficient

Largest diff. peak and hole
2106912

s18uh2

$\mathrm{C}_{24} \mathrm{H}_{27} \mathrm{~K} \mathrm{O}_{2}$

386.55

150.01(10) K

$1.54184 \AA$

Triclinic

P-1

$a=9.3187(2) \AA$ $\alpha=91.443(2)^{\circ}$

$\mathrm{b}=10.3999(3) \AA$

$\beta=103.837(2)^{\circ}$

$c=12.2070(2) \AA$

$\gamma=114.291(2)^{\circ}$

$1036.74(4) \AA^{3}$

2

$1.238 \mathrm{Mg} / \mathrm{m}^{3}$

$2.350 \mathrm{~mm}^{-1}$

412

$0.300 \times 0.200 \times 0.120 \mathrm{~mm}^{3}$

3.766 to $73.134^{\circ}$

$-11<=\mathrm{h}<=11,-12<=\mathrm{k}<=12,-15<=\mid<=15$

19692

$4136[R$ (int) $=0.0270]$

$100.0 \%$

Semi-empirical from equivalents

1.00000 and 0.76236

Full-matrix least-squares on $\mathrm{F}^{2}$

4136 / 0 / 244

1.043

$R 1=0.0340, w R 2=0.0904$

$R 1=0.0346, w R 2=0.0910$

$\mathrm{n} / \mathrm{a}$

0.487 and -0.300 e..$^{-3}$ 


\section{f. Crystal data and structure refinement for pyridine-coordinated dipotassium 1,3,4,6-tetraphenylpentalenide}

CCDC number
Identification code
Empirical formula
Formula weight
Temperature
Wavelength
Crystal system
Space group
Unit cell dimensions

Volume

Z

Density (calculated)

Absorption coefficient

$\mathrm{F}(000)$

Crystal size

Theta range for data collection

Index ranges

Reflections collected

Independent reflections

Completeness to theta $=67.684^{\circ}$

Absorption correction

Max. and min. transmission

Refinement method

Data / restraints / parameters

Goodness-of-fit on $\mathrm{F}^{2}$

Final $R$ indices [ $\mid>2 \operatorname{sigma}(I)]$

$R$ indices (all data)

Extinction coefficient

Largest diff. peak and hole
2106913

s19uh7

$\mathrm{C}_{62} \mathrm{H}_{52} \mathrm{~K}_{2} \mathrm{~N}_{6}$

959.29

$150.00(10) \mathrm{K}$

$1.54184 \AA$

Monoclinic

$\mathrm{C} 2 / \mathrm{c}$

$a=15.6676(2) \AA \quad \alpha=90^{\circ}$

$b=14.14470(10) \AA \quad \beta=108.1770(10)^{\circ}$

$\mathrm{c}=25.0883(3) \AA \quad \gamma=90^{\circ}$

$5282.45(10) \AA^{3}$

4

$1.206 \mathrm{Mg} / \mathrm{m}^{3}$

$1.927 \mathrm{~mm}^{-1}$

2016

$0.336 \times 0.291 \times 0.168 \mathrm{~mm}^{3}$

3.709 to $72.583^{\circ}$

$-14<=h<=18,-17<=k<=17,-30<=\mid<=31$

44192

$5189[R($ int $)=0.0361]$

$99.9 \%$

Semi-empirical from equivalents

1.00000 and 0.38346

Full-matrix least-squares on $\mathrm{F}^{2}$

5189 / 0 / 316

1.028

$\mathrm{R} 1=0.0348, \mathrm{wR} 2=0.0884$

$R 1=0.0370, w R 2=0.0897$

$\mathrm{n} / \mathrm{a}$

0.324 and -0.353 e. $\AA^{-3}$ 